\title{
Observation of interstellar lithium in the low-metallicity Small Magellanic Cloud
}

\author{
J. Christopher Howk ${ }^{1}$, Nicolas Lehner ${ }^{1}$, Brian D. Fields ${ }^{2,3}$ \& Grant J. Mathews ${ }^{1}$ \\ ${ }^{1}$ Department of Physics, Center for Astrophysics, University of Notre Dame, Notre Dame, IN 46556, USA \\ ${ }^{2}$ Department of Astronomy, University of Illinois at Urbana-Champaign, Urbana, IL 61801, USA \\ ${ }^{3}$ Department of Physics, University of Illinois at Urbana-Champaign, Urbana, IL 61801, USA
}

The primordial abundances of light elements produced in the standard theory of Big Bang nucleosynthesis $(\mathrm{BBN})$ depend only on the cosmic ratio of baryons to photons, a quantity inferred from observations of the microwave background. ${ }^{1}$ The predicted ${ }^{2-4}$ primordial ${ }^{7} \mathrm{Li}$ abundance is four times that measured in the atmospheres of Galactic halo stars.$^{5 \cdot 7]}$ This discrepancy could be caused by modification of surface lithium abundances during the stars' lifetimes $^{8}$ or by physics beyond the Standard Model that impacts early nucleosynthesis. ${ }^{9} \cdot 10$ The lithium abundance of low-metallicity gas provides an alternative constraint on the primordial abundance and cosmic evolution of lithium ${ }^{11}$ that is not susceptible to the in situ modifications that may affect stellar atmospheres. Here we report observations of interstellar ${ }^{7} \mathrm{Li}$ in the low-metallicity gas of the Small Magellanic Cloud, a nearby galaxy with one quarter of the Sun's metallicity. The present-day ${ }^{7} \mathrm{Li}$ abundance in the Small Magellanic Cloud is nearly equal to the BBN predictions, severely constraining the amount of possible subsequent enrichment of the gas by stellar and cosmic ray nucleosynthesis. Our measurements can be reconciled with standard BBN with an extremely fine-tuned depletion of stellar Li with metallicity. They are also consistent with non-standard BBN.

We obtained high resolution spectra $(R \approx 70,000)$ of the star Sk 143 (AzV 456), an O9.5 Ib star in the Small Magellanic Cloud (SMC), using UVES ${ }^{12}$ on the 8.2-m VLT (observational details are given in the Supplementary Information). The sight line to this star was chosen for observation because it shows significant absorption from neutral atoms and molecules $\frac{13}{15}$ and a weak interstellar radiation field, $\frac{14}{14}$ all of which favor the presence of neutral lithium, Li I. Li I absorption is clearly detected along this sight line (Figure 1). 
The derivation of the total $\mathrm{Li} / \mathrm{H}$ abundance in the interstellar medium (ISM) requires large corrections for ionization, since $N(\mathrm{Li}) \approx N(\mathrm{Li} \mathrm{II}) \gg N(\mathrm{Li} \mathrm{I})$, and for the incorporation of Li into interstellar dust grains .19 Our first approach to these corrections uses observations of adjacent ionization states of other metals, in this case $\mathrm{Ca}$ and $\mathrm{Fe}$, to estimate the amount of unseen gas phase lithium. Assuming ionization balance and only atomic processes, the ratio $N(\mathrm{Li}$ II $) / N(\mathrm{Li} \mathrm{I}) \propto$ $N(\mathrm{Ca}$ II $) / N(\mathrm{Ca} \mathrm{I})$ or $\propto N(\mathrm{Fe}$ II $) / N(\mathrm{Fe} \mathrm{I})$, where the constant of proportionality involves the ratios of ionization rates and recombination coefficients for the elements in question. ${ }^{19 \mid 20}$ The ratio of ${ }^{7} \mathrm{Li}$ I to total hydrogen in the SMC is $\log \left[N\left({ }^{7} \mathrm{Li} \mathrm{I}\right) / N(\mathrm{H})\right]=-11.17 \pm 0.04$ (all uncertainties are $1 \sigma$ unless noted), where $N(\mathrm{H}) \equiv N(\mathrm{H} \mathrm{I})+2 N\left(\mathrm{H}_{2}\right)$. Applying ionization corrections derived from $\mathrm{Ca}$ and Fe yields logarithmic abundances $A\left({ }^{7} \mathrm{Li}\right) \equiv \log \left[N\left({ }^{7} \mathrm{Li}\right) / N(\mathrm{H})\right]+12=2.79 \pm 0.11$ and $3.01 \pm 0.12$. These calculations do not include more complicated (and uncertain) effects such as grain-assisted recombination, $\sqrt[20]{21}$ nor do they correct for dust depletion.

Our second approach uses the observation ${ }^{19}$ that $N\left({ }^{7} \mathrm{Li} \mathrm{I}\right) / N(\mathrm{~K} \mathrm{I})$ along sight lines through the Milky Way is nearly constant (with new determinations giving consistent results $\frac{18}{22}$ ). When a differential ionization correction is applied, ${ }^{7} \mathrm{Li} / \mathrm{K}$ in the Milky Way ISM is consistent with the solar system ratio. Thus, ${ }^{7} \mathrm{Li}$ and $\mathrm{K}$ appear to have very similar ionization and dust depletion behaviors, and ${ }^{7} \mathrm{Li} \mathrm{I} / \mathrm{K}$ I gives a good measure of the total (gas+dust phase) ${ }^{7} \mathrm{Li} / \mathrm{K} \cdot{ }^{\left[1{ }^{192}\right.}$ We measure $\log \left[N\left({ }^{7} \mathrm{Li} \mathrm{I}\right) / N(\mathrm{~K} \mathrm{I})\right]=-2.27 \pm 0.03$ in the SMC, in agreement with the Galactic relationship 18 22 Applying an ionization correction of $+0.54 \pm 0.08 \mathrm{dex}^{1920}$ gives $\log \left[N\left({ }^{7} \mathrm{Li}\right) / N(\mathrm{~K})\right]=-1.78 \pm$ 0.09. With the solar system ratio $\log \left({ }^{7} \mathrm{Li} / \mathrm{K}\right)_{\odot}=-1.82 \pm 0.05$ derived from meteorites, ${ }^{[23}$ we find $\left[{ }^{7} \mathrm{Li} / \mathrm{K}\right]_{\mathrm{SMC}} \equiv \log \left[N\left({ }^{7} \mathrm{Li}\right) / N(\mathrm{~K})\right]-\log \left({ }^{7} \mathrm{Li} / \mathrm{K}\right)_{\odot}=+0.04 \pm 0.10$. The ratio of ${ }^{7} \mathrm{Li}$ to metal nuclei in the SMC is consistent with that found in the solar system and the Milky Way ISM ${ }^{19}$ : $\left({ }^{7} \mathrm{Li} / \mathrm{K}\right)_{\mathrm{SMC}} \approx\left({ }^{7} \mathrm{Li} / \mathrm{K}\right) \odot$

Although the ionization and depletion characteristics of S I are not as well tied to those of $\mathrm{Li} \mathrm{I},{ }^{20}$ a similar approach using S I yields $\left[{ }^{7} \mathrm{Li} / \mathrm{S}\right]_{\mathrm{SMC}}=-0.26 \pm 0.11$. The sub-solar ratio is consistent with a modest $(0.3$ dex $)$ depletion of Li and $\mathrm{K}$ onto dust in the ISM ${ }^{18}$ relative to $\mathrm{S}$.

We estimate $A\left({ }^{7} \mathrm{Li}\right)$ by scaling ${ }^{7} \mathrm{Li} / \mathrm{K}$ to $\mathrm{Li} / \mathrm{H}: A\left({ }^{7} \mathrm{Li}\right)_{\mathrm{SMC}}=A\left({ }^{7} \mathrm{Li}\right)_{\odot}+[\mathrm{Fe} / \mathrm{H}]_{\mathrm{SMC}}+[\mathrm{K} / \mathrm{Fe}]_{\mathrm{SMC}}+$ 
$\left[{ }^{7} \mathrm{Li} / \mathrm{K}\right]_{\mathrm{SMC}}$. We adopt $\left[{ }^{7} \mathrm{Li} / \mathrm{K}\right]_{\mathrm{SMC}}$ from above, the meteoritic $A\left({ }^{7} \mathrm{Li}\right) \odot=3.23 \pm 0.05,{ }^{23}$ with a mean present-day SMC metallicity $[\mathrm{Fe} / \mathrm{H}]_{\mathrm{SMC}}=-0.59 \pm 0.06$ and an $\mathrm{SMC} \mathrm{K} / \mathrm{Fe}$ abundance $[\mathrm{K} / \mathrm{Fe}]_{\mathrm{SMC}} \equiv+0.00 \pm 0.10$ (the last two discussed in the Supplementary Information). This yields $A\left({ }^{7} \mathrm{Li}\right)_{\mathrm{SMC}}=2.68 \pm 0.16$. Similarly scaling the ${ }^{7} \mathrm{Li} / \mathrm{S}$ result gives $2.38 \pm 0.17$.

Most previous observational constraints on the primordial Li abundance have relied on measurements of atmospheric abundances in low-metallicity Galactic stars. Our first detection of interstellar lithium beyond the Milky Way opens a new window on the lithium problem. While there are significant uncertainties associated with ionization and dust effects, as demonstrated by the significant spread in $A\left({ }^{7} \mathrm{Li}\right)_{\mathrm{SMC}}$ values, these are largely independent of the uncertainties that might affect stellar measurements of the primordial lithium abundance. Our recommended absolute abundance is $A\left({ }^{7} \mathrm{Li}\right)_{\mathrm{SMC}}=2.68 \pm 0.16$, or $\left({ }^{7} \mathrm{Li} / \mathrm{H}\right)_{\mathrm{SMC}}=(4.8 \pm 1.8) \times 10^{-10}$, derived from ${ }^{7} \mathrm{Li} / \mathrm{K}$. This is compared to stellar ${ }^{7} \mathrm{Li}$ abundances ${ }^{616}$ at different metallicities in Figure 2, Our best estimate overlaps the prediction from standard BBN using the baryonic density deduced from the 5-year WMAP data, ${ }^{1} A\left({ }^{7} \mathrm{Li}\right)=2.72 \pm 0.06$ (95\% c.1.) ${ }^{3}$ although this leaves little room for the post-BBN chemical evolution, $25 \mid 26]$ i.e., the contribution of freshly-synthesized Li to the ISM by stellar and cosmic ray nucleosynthesis (see representative models ${ }^{25}$ in Figure 2). Our estimate of $A\left({ }^{7} \mathrm{Li}\right)_{\mathrm{SMC}}$ is also consistent with the upper envelope of $\mathrm{Li}$ abundances in Milky Way thin disc stars (Figure 2). 16

However, given the uncertainties in scaling to $A\left({ }^{7} \mathrm{Li}\right)_{\mathrm{SMC}}$, the stronger result is our measurement $\left[{ }^{7} \mathrm{Li} / \mathrm{K}\right]_{\mathrm{SMC}}=+0.04 \pm 0.10$. We compare $\left[{ }^{7} \mathrm{Li} / \mathrm{K}\right]_{\mathrm{SMC}}$ with measurements ${ }^{61623}$ of $\left[{ }^{7} \mathrm{Li} / \mathrm{Fe}\right]$ and chemical evolution models ${ }^{25}$ in Figure 3. The stars show a rapid decrease in $\left[{ }^{7} \mathrm{Li} / \mathrm{Fe}\right]$ with increasing metallicity until $[\mathrm{Fe} / \mathrm{H}] \approx-1$, at which point the Li abundance increases roughly in lockstep with Fe such that disc stars have a nearly-constant $\left[{ }^{7} \mathrm{Li} / \mathrm{Fe}\right]$ ratio similar to that found in the solar system. Our measurement of the present day ${ }^{7} \mathrm{Li}$-to-metal ratio in the SMC is in agreement with the nearly constant values found in the atmospheres of Milky Way disc stars $(-1 \lesssim[\mathrm{Fe} / \mathrm{H}] \lesssim 0)$, most of which formed $>4$ Gyr ago, with the solar system, and the modern-day Milky Way ISM 19

Both the thin disc stars and our SMC measurements are below standard BBN predictions with 
reasonable assumptions about post-BBN production, although it is often assumed these stars have had significant depletion of their surface Li abundance. ${ }^{[25}$ Taken at face value, the consistency of our SMC measurement with the $\left[{ }^{7} \mathrm{Li} / \mathrm{Fe}\right]$ for those stars calls this assumption into question. While the models in Figures 2 and 3 are imprecise given the uncertain Li yields from stellar sources, they illustrate the tension between standard BBN predictions and our measurements if there is any post-BBN Li production. This tension can be relieved if a metallicity-dependent depletion of $\mathrm{Li}$ in stellar atmospheres is fine tuned in such a way that it is very strong below $[\mathrm{Fe} / \mathrm{H}] \approx$ $[\mathrm{Fe} / \mathrm{H}]_{\mathrm{SMC}}=-0.6$ (to create the Spite plateau and avoid overproducing Li in the SMC ISM) and negligible at or above the SMC metallicity, conspiring to create a constant $\left[{ }^{7} \mathrm{Li} / \mathrm{Fe}\right]$ ratio above $[\mathrm{Fe} / \mathrm{H}] \approx-1$. Alternatively, non-standard BBN scenarios can be invoked to allow for a lower primordial Li abundance. $\underline{4} 27$

If non-standard Li production occurs in the BBN epoch, many such models predict excess ${ }^{6} \mathrm{Li}$ compared with standard BBN. The only known source of post-Big Bang ${ }^{6} \mathrm{Li}$ is production via cosmic ray interactions with ISM particles. Excess ${ }^{6} \mathrm{Li}$ at the metallicity of the SMC would support non-standard production mechanisms, either in the BBN epoch ${ }^{10}$ or through the interaction of pregalactic cosmic rays with intergalactic helium. ${ }^{28}$ Measurements of ${ }^{6} \mathrm{Li}$ in stellar atmospheres are extremely difficult since the stellar line broadening is well in excess of the isotope shift. However, the ${ }^{7} \mathrm{Li}$ I doublet is well-separated in our data due to the very low broadening in the cool ISM probed by Li I absorption. Our best fit to the SMC Li I absorption gives $\left({ }^{6} \mathrm{Li} /{ }^{7} \mathrm{Li}\right)_{\mathrm{SMC}}=0.13 \pm$ 0.05 (see Supplementary Information and Figure 1), giving a formal limit to the isotopic ratio in the SMC of $\left({ }^{6} \mathrm{Li} /{ }^{7} \mathrm{Li}\right)_{\mathrm{SMC}}<0.28(3 \sigma)$. With higher signal-to-noise and resolution it should be possible to lower the limits for the interstellar isotope ratio in the SMC to a point that they provide constraints on non-standard BBN models. This approach has the advantage that ionization and dust depletion effects are not important for comparing the two isotopes of $\mathrm{Li},{ }^{[17}$ making ${ }^{6} \mathrm{Li} /{ }^{7} \mathrm{Li}$ a powerful diagnostic of nucleosynthesis and non-standard evolution of $\mathrm{Li}$ abundances. 
1. Dunkley, J., et al. Five-Year Wilkinson Microwave Anisotropy Probe Observations: Likelihoods and Parameters from the WMAP Data. Astrophys. J. Suppl., 180, 306-329 (2009)

2. Steigman, G. Primordial Nucleosynthesis in the Precision Cosmology Era. Ann. Rev. Nuc. Part. Science, 57, 463-491 (2007)

3. Cyburt, R. H., Fields, B. D., \& Olive, K. A. An update on the big bang nucleosynthesis prediction for ${ }^{7} \mathrm{Li}$ : the problem worsens. Journal of Cosmology and Astro-Particle Physics, 11, $012(2008)$

4. Fields, B. D. The Primordial Lithium Problem. Ann. Rev. Nuc. Part. Science 61, 47-68 (2011)

5. Spite, M., \& Spite, F. Lithium abundance at the formation of the Galaxy. Nature, 297, 483-485 (1982)

6. Sbordone, L., et al. The metal-poor end of the Spite plateau. 1: Stellar parameters, metallicities and lithium abundances. Astron. \& Astrophys., 522, A26 (2010)

7. Meléndez, J., Casagrande, L., Ramírez, I., Asplund, M., \& Schuster, W. J. Observational evidence for a broken Li Spite plateau and mass-dependent Li depletion. Astron. \& Astrophys., 515, L3-L7 (2010)

8. Korn, A. J., Grundahl, F., Richard, O., Barklem, P. S., Mashonkina, L., Collet, R., Piskunov, N., \& Gustafsson, B. A probable stellar solution to the cosmological lithium discrepancy. Nature, 442, 657-659 (2006)

9. Jedamzik, K. Did something decay, evaporate, or annihilate during big bang nucleosynthesis?. Phys. Rev. D, 70, 063524-(2004)

10. Pospelov, M., \& Pradler, J. Big Bang Nucleosynthesis as a Probe of New Physics. Ann. Rev. Nuc. Part. Science, 60, 539-568 (2010)

11. Prodanović, T., \& Fields, B. D. Probing Primordial and Pre-Galactic Lithium with HighVelocity Clouds. Astrophys. J., 616, L115-L118 (2004) 
12. Dekker, H., D’Odorico, S., Kaufer, A., Delabre, B., \& Kotzlowski, H. Design, construction, and performance of UVES, the echelle spectrograph for the UT2 Kueyen Telescope at the ESO Paranal Observatory. Proc. SPIE, 4008, 534-545 (2000)

13. Cox, N. L. J., et al. Interstellar gas, dust and diffuse bands in the SMC. Astron. \& Astrophys., 470, 941-955 (2007)

14. Welty, D. E., Federman, S. R., Gredel, R., Thorburn, J. A., \& Lambert, D. L. VLT UVES Observations of Interstellar Molecules and Diffuse Bands in the Magellanic Clouds. Astrophys. J. Suppl., 165, 138-172 (2006)

15. Cartledge, S. I. B., et al. FUSE Measurements of Far-Ultraviolet Extinction. II. Magellanic Cloud Sight Lines. Astrophys. J., 630, 355-367 (2005)

16. Lambert, D. L., Reddy, B. E. Lithium abundances of the local thin disc stars Mon. Not. R. Astron. Soc., 349, 757-767 (2004)

17. Kawanomoto, S., et al. The New Detections of ${ }^{7} \mathrm{Li} /{ }^{6} \mathrm{Li}$ Isotopic Ratio in the Interstellar Media. Astrophys. J., 701, 1506-1518 (2009)

18. Knauth, D. C., Federman, S. R., \& Lambert, D. L. An Ultra-high-Resolution Survey of the Interstellar ${ }^{6} \mathrm{Li} /{ }^{7} \mathrm{Li}$ Isotope Ratio in the Solar Neighborhood. Astrophys. J., 586, 268-285 (2003)

19. Steigman, G. Cosmic Lithium: Going Up or Coming Down?. Astrophys. J., 457, 737-742 (1996)

20. Welty, D. E., Hobbs, L. M., \& Morton, D. C. High-Resolution Observations of Interstellar Ca I Absorption-Implications for Depletions and Electron Densities in Diffuse Clouds. Astrophys. J. Suppl., 147, 61-96 (2003)

21. Weingartner, J. C., \& Draine, B. T. Electron-Ion Recombination on Grains and Polycyclic Aromatic Hydrocarbons. Astrophys. J., 563, 842-852 (2001) 
22. Welty, D. E., \& Hobbs, L. M. A High-Resolution Survey of Interstellar K I Absorption. Astrophys. J. Suppl., 133, 345-393 (2001)

23. Asplund, M., Grevesse, N., Sauval, A. J., \& Scott, P. The Chemical Composition of the Sun. Ann. Rev. Astron. Astrophys., 47, 481-522 (2009)

24. Ryan, S. G., Kajino, T., Beers, T. C., Suzuki, T. K., Romano, D., Matteucci, F., \& Rosolankova, K. Abundances and Evolution of Lithium in the Galactic Halo and Disk. Astrophys. J., 549, $55-71(2001)$

25. Prantzos, N. Production and evolution of Li, Be and B isotopes in the Galaxy Astron. \& Astrophys., in press (arXiv:1203.5662) (2012)

26. Romano, D., Tosi, M., Matteucci, F., \& Chiappini, C. Light element evolution resulting from WMAP data. Mon. Not. R. Astron. Soc., 346, 295-303 (2003)

27. Iocco, F., Mangano, G., Miele, G., Pisanti, O., \& Serpico, P. D. Primordial nucleosynthesis: From precision cosmology to fundamental physics. Phys. Reports, 472, 1-76 (2009)

28. Suzuki, T. K., \& Inoue, S. Cosmic-Ray Production of ${ }^{6} \mathrm{Li}$ by Structure Formation Shocks in the Early Milky Way: A Fossil Record of Dissipative Processes during Galaxy Formation. Astrophys. J., 573, 168-173 (2002)

Supplementary Information is linked to the online version of the paper at www.nature.com/nature.

Acknowledgements We thank the European Southern Observatory for granting us time for this project as part of proposal 382.B-0556. We also thank A. Fox and H. Sana for helpful discussions about the UVES data and A. Korn, P. Molaro, T. Prodanovic, D. Romano, and D. Welty with helpful input on the project that improved the paper.

Author Contributions All authors participated in the interpretation and commented on the manuscript. J.C.H. led the project and was responsible for the text of the paper. 
Author Information Correspondence and requests for materials should be addressed to J.C.H. (email: jhowk@nd.edu). 

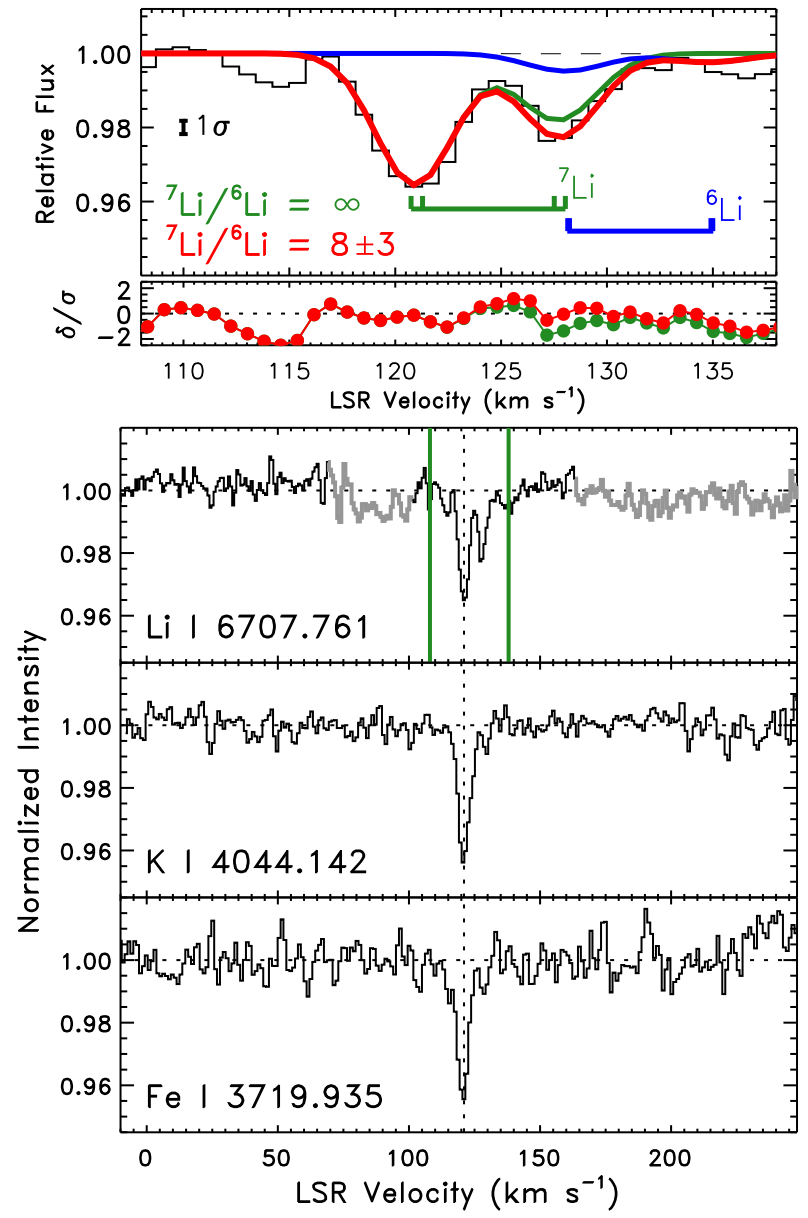

Figure 1: Interstellar absorption by several neutral species seen toward Sk 143. Normalized interstellar absorption profiles from UVES plotted versus Local Standard of Rest (LSR) velocity and profile fit of the Li I absorption. The empirically-determined SNR is $\sim 275$ per pixel (5 pixels per resolution element) for the Li I observations. The full set of optical and ultraviolet absorption profiles seen toward this star and column densities measured from these are given in the Supplementary Information. The lower three panels show the profiles of Li I, K I, and Fe I; the SMC cloud bearing Li I at $v_{\mathrm{LSR}} \approx+121 \mathrm{~km} \mathrm{~s}^{-1}$ is marked with the dashed line. The grey regions near Li I are possibly contaminated by diffuse interstellar bands or residual fringing, which may extend into the region containing Li absorption. The effects on the ${ }^{7} \mathrm{Li}$ I columns are within the quoted uncertainties. The Li I absorption is composed of (hyper)fine structure components of both ${ }^{7} \mathrm{Li} \mathrm{I}$ and ${ }^{6} \mathrm{Li} \mathrm{I}$ (shown respectively by the green and blue ticks in the top panel). The strong line along of ${ }^{7} \mathrm{Li}$ I is detected with $\sim 16 \sigma$ significance in the ISM of the SMC. A model fit to the Li I absorption complex is shown in the top panel (see Supplementary Information), with the fit residuals shown immediately below (normalized to the local error array). The free parameters for the fit are the polynomial coefficients for the stellar continuum, the central velocity, Doppler parameter (b-value), and column densities of ${ }^{7} \mathrm{Li} \mathrm{I}$ and ${ }^{6} \mathrm{Li}$ I for the interstellar cloud. The red curve shows the best fit joint fit including both ${ }^{7} \mathrm{Li} \mathrm{I}$ and ${ }^{6} \mathrm{Li} \mathrm{I}$, shown in green and blue, respectively. The best fit isotopic ratio is $N\left({ }^{6} \mathrm{Li} \mathrm{I}\right) / N\left({ }^{7} \mathrm{Li} \mathrm{I}\right)=0.13 \pm 0.05$ (68\% c.l.), consistent with the presence of ${ }^{6} \mathrm{Li}$ along the sight line, although below the $3 \sigma$ detection threshold. 


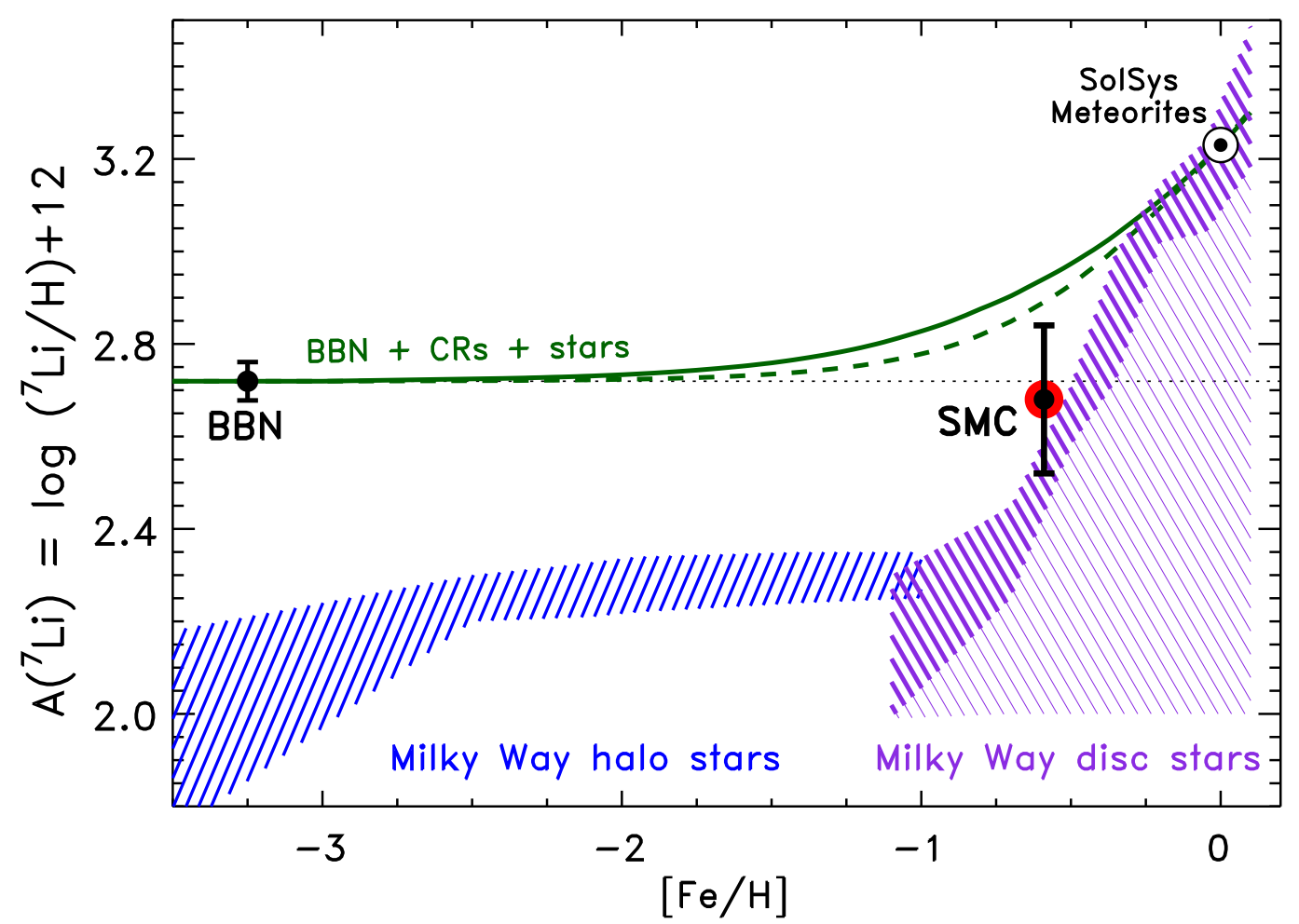

Figure 2: Estimates of the lithium abundance in the SMC interstellar medium and in several different environments. Our best estimate for interstellar gas+dust phase abundance of $A\left({ }^{7} \mathrm{Li}\right)$ in the $\mathrm{SMC}$ is shown as the red circle with black core derived from the ${ }^{7} \mathrm{Li} \mathrm{I} / \mathrm{K} \mathrm{I}$ ratio. The present day metallicity of the SMC from early-type stars is $[\mathrm{Fe} / \mathrm{H}]=-0.59 \pm 0.06$. (All uncertainties are $1 \sigma$.) The point marked $\mathrm{BBN}$ and dotted horizontal line show the primordial abundance predicted by standard $\mathrm{BBN}{ }^{[3}$ The green curves show recent models ${ }^{25}$ for post-BBN ${ }^{7} \mathrm{Li}$ nucleosynthesis due to cosmic rays (CRs) and stars. By adjusting the yields from low-mass stars, the models are forced to match the solar system meteoritic abundance ${ }^{23}$ (see Supplementary Information). The solid and dashed lines correspond to models $A$ and $\mathrm{B}^{25}$ which respectively include or not a presumed contribution to ${ }^{7} \mathrm{Li}$ from core-collapse supernovae. The blue hatched area shows the range of abundances derived for Population II stars in the Galactic halo, ${ }^{6}$ with the "Spite plateau" in this sample at $A\left({ }^{7} \mathrm{Li}\right)_{\mathrm{PopII}} \approx 2.10 \pm 0.10{ }^{[6}$ The violet hatched region shows the range of measurements seen in Galactic thin disk stars, where the thicker lines denote the six most Li-rich stars in a series of eight metallicity bins. ${ }^{16}$ The selection of thin disk stars includes objects over a range of masses and temperatures, including stars that are expected to have destroyed a fair fraction of their Li. Thus, the upper envelope of the distribution represents the best estimate of the intrinsic ISM Li abundance at the epoch of formation for those stars, and the thicker dashed lines for the thin disk sample are most appropriate for comparison with the SMC value. The most Li-rich stars in the Milky Way thin disc ${ }^{16}$ within 0.1 dex of the SMC metallicity give $A\left({ }^{7} \mathrm{Li}\right)_{\mathrm{MW}}=2.54 \pm 0.05$, consistent with our estimate $A\left({ }^{7} \mathrm{Li}\right)_{S M C}=2.68 \pm 0.16$. 


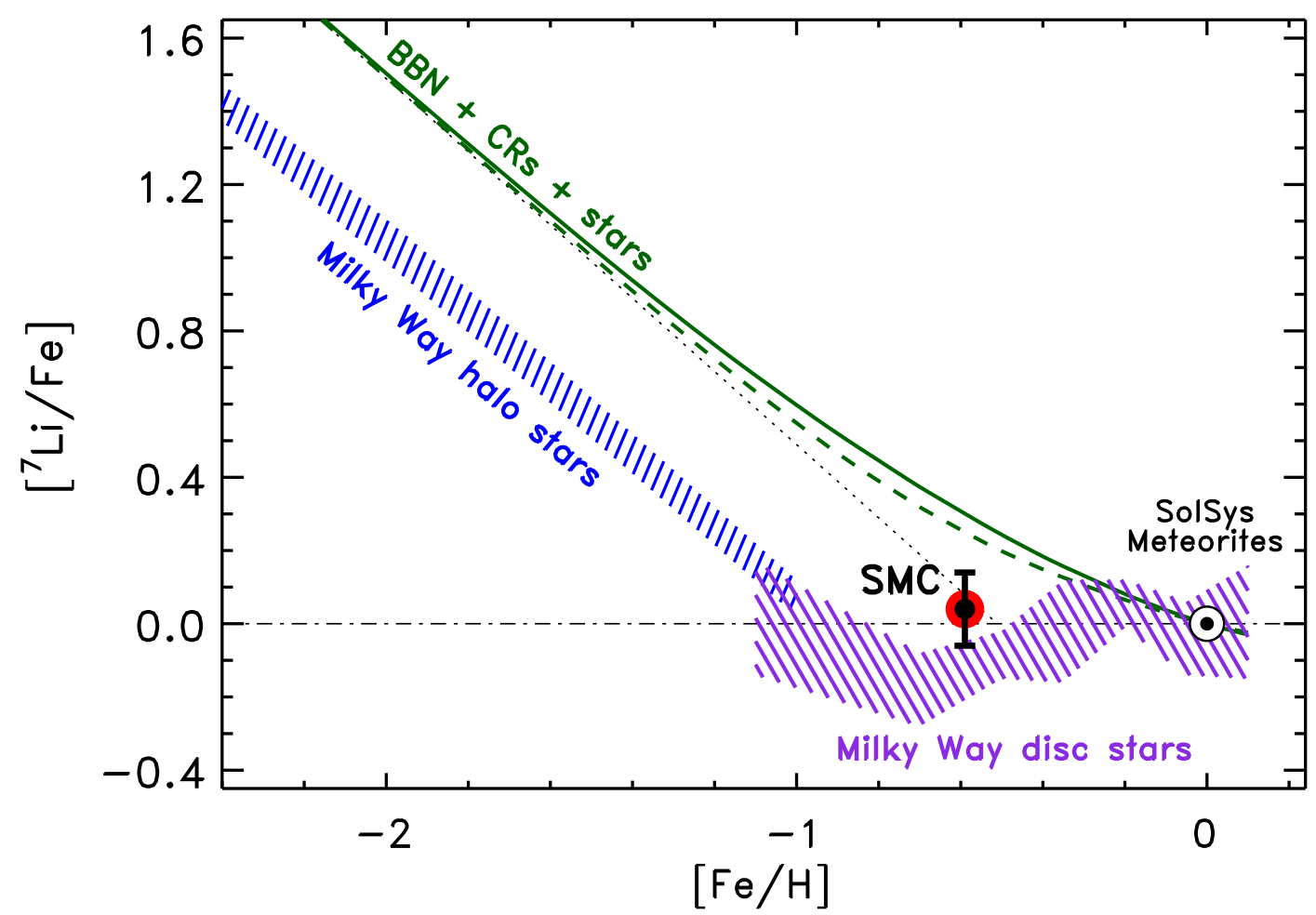

Figure 3: Estimates of $\mathrm{Li} / \mathrm{Fe}$ in the SMC interstellar medium and in several different environments. The SMC value is derived from the ${ }^{7} \mathrm{Li} \mathrm{I} / \mathrm{K}$ I ratio. At low metallicities $([\mathrm{Fe} / \mathrm{H}] \lesssim-1)$, stellar measurements ${ }^{6}$ trace the build-up of $\mathrm{Fe}$ with a constant $\mathrm{Li}$ abundance along the Spite plateau. At higher metallicities, disc star abundances ${ }^{16}$ show a turnover to roughly constant $\left[{ }^{7} \mathrm{Li} / \mathrm{Fe}\right]$ at values consistent with the solar system/meteoritic value ${ }^{23}$ (shown as the dash-dotted line). Our SMC estimate is consistent with the solar system and disc star abundances in this region of relatively constant ${ }^{7} \mathrm{Li} / \mathrm{Fe}$ abundances, with $\left[{ }^{7} \mathrm{Li} / \mathrm{Fe}\right]_{\mathrm{SMC}}=+0.04 \pm 0.14$ for $[\mathrm{K} / \mathrm{Fe}]_{\mathrm{SMC}}=0.0 \pm 0.10$ (Supplemental Information). The most Li-rich disc stars within 0.1 dex of the SMC metallicity have a mean $\left\langle\left[{ }^{7} \mathrm{Li} / \mathrm{Fe}\right]\right\rangle=-0.13 \pm 0.05$. (All uncertainties are $1 \sigma$.$) The green curves show the chemical evolution model { }^{25}$ as in Figure 2, while the dotted line shows the behavior of $\left[{ }^{7} \mathrm{Li} / \mathrm{Fe}\right]$ for the standard BBN primordial abundance with no subsequent evolution of ${ }^{7} \mathrm{Li}$. The relative uniformity of the stellar ${ }^{7} \mathrm{Li} / \mathrm{Fe}$ abundances at $[\mathrm{Fe} / \mathrm{H}] \gtrsim-1$ could be caused by a delicate balance of $\mathrm{Li}$ and $\mathrm{Fe}$ production and metallicity-dependent $\mathrm{Li}$ astration (not ruled out given the changes in mean age and mass potentially present in the sample ${ }^{\sqrt{16}}$ ). However, the agreement of the $\left[{ }^{7} \mathrm{Li} / \mathrm{Fe}\right]$ ratio seen in these old stars (ages $>4 \mathrm{Gyl}^{16}$ ) and in the present-day interstellar medium of the SMC suggests little change in the stellar abundances for metallicities $[\mathrm{Fe} / \mathrm{H}] \gtrsim-0.6$ through the solar metallicity. To bring the stellar and SMC interstellar abundances into agreement with standard BBN predictions requires a delayed injection of significant ${ }^{7} \mathrm{Li}$ from stellar production mechanisms as well as vigorous depletion of stellar surface ${ }^{7} \mathrm{Li}$ abundances at metallicities just below that of the SMC. 


\section{Supplementary Information}

\section{Observations and Data Reduction}

We use spectroscopic data from two instruments in our analysis: ground-based optical observations from the Ultraviolet Echelle Spectrograph (UVES) ${ }^{12}$ on ESO's Very Large Telescope (UT-2), and space-based ultraviolet observations from the Space Telescope Imaging Spectrograph (STIS) onboard the Hubble Space Telescope.

UVES Data The UVES data presented here were taken in service mode on 2008 September 25 under the program 382.B-0556(A). The observations consist of 10 exposures of 2850 seconds each. We used the $0^{\prime \prime} .7$ slit, feeding the light to dichroic \#2. The grating central wavelengths were 3,900 $\AA$ in the blue and 7,600 $\AA$ in the red. No binning was done on the chips. The data were taken through thin clouds and with seeing ranging from $\approx 1$." 0 to $1^{\prime \prime} .5$.

The data were reduced using the UVES pipeline provided by ESO. In order to achieve high signal-to-noise, we used the pixel-by-pixel flat field correction, setting "FFMETHOD=pixel" in the reduction pipeline and using optimal extraction of the data. The individual exposures were corrected for heliocentric motion and coadded, weighted by the variance. The signal-to-noise ratio $(S N R)$ varies from $S N R \sim 150$ per pixel in the far blue (e.g., near Ti II 3,383) to $\sim 300$ in some regions of the spectrum (e.g., the $\mathrm{Ca}$ I and $\mathrm{CH}^{+}$transitions near 4,200 $\AA$ ). Near the Li I transition at $\lambda \approx 6,710 \AA$ we measure $S N R \sim 275$.

UVES Line Spread Function: The width of the UVES line spread function (LSF) is important for deriving accurate fit parameters for the blended isotopes of Li. While ultimately the column density of Li I is not strongly changed by small changes in the LSF width, the $b$-values are somewhat sensitive to the LSF width. The UVES pipeline produces estimates of the width of the LSF on the basis of observations of ThAr lamps taken for wavelength calibration. For the blue CCD data, the ESO quality-control summaries available on-line suggest the resolution for our set-up during this time is $R \approx 66,000$ or $\Delta v \approx 4.54 \mathrm{~km} \mathrm{~s}^{-1}$, which is appropriate for roughly the month surrounding our observations. The ThAr lines in our own calibration observations yield a value 
indistinguishable from this.

On the red chip (REDL), notably in the vicinity of the Li I lines near 6,710 , the mean FWHM of these lines from our calibration data is $4.47 \mathrm{~km} \mathrm{~s}^{-1}$ for the 38 lines in the range $6,700 \AA<\lambda<6,720 \AA$ and $4.42 \mathrm{~km} \mathrm{~s}^{-1}$ for the 225 lines over the broader range $6,650 \AA<\lambda<$ $6,770 \AA$. These correspond to a resolution $R \approx 68,000$.

However, in the course of fitting the Li I blend, adopting this resolution seemed to underpredict the cores of the lines and provide too much absorption immediately outside of the cores, suggesting the adopted LSF may be too broad. D. Welty kindly provided us with Na I 5,889/5,895 observations of the Sk 143 sight line taken with the CES spectrograph on the ESO 3.6-m telescope with a resolution $\Delta v=1.35 \mathrm{~km} \mathrm{~s}^{-1}$ (FWHM) or $R \approx 222,000$. These data were presented in Welty et al ${ }^{14}$ We derived the best LSF breadth for the current UVES red CCD data by comparing smoothed CES data with the UVES observations of these absorption lines, using the $\chi^{2}$ as an estimate of the goodness of fit. The free parameters of the model in this case were the breadth of the smoothing kernel (assumed Gaussian) and the background offset of the CES observations (for which the original background subtraction was derived by matching equivalent widths of the $\mathrm{Na}$ I lines with earlier UVES data $\left.{ }^{(14}\right)$.

The final result of this approach yields a best fit LSF breadth of $\Delta v=4.03 \pm 0.08 \mathrm{~km} \mathrm{~s}^{-1}$ (FWHM) or resolution $R=74,300$, which we adopt in fitting the Li I blend. The difference between our derived LSF and the values from the ThAr emission lines is likely due to the manner in which the light fills (or not) the slit during the observations. We caution that we assume in our fitting a single Gaussian LSF; the true LSF may be more complex than this. However, we have tested the impact of the LSF on our measurement of both the ${ }^{7} \mathrm{Li}$ I column and isotopic ratio (see below), and find the results robust to even $5 \sigma$ variations in the LSF breadth. This LSF breadth likely only applies to the data taken with the REDL chip. LSF changes with wavelength and detector are to be expected. However, the breadth of the LSF for the data taken with the blue side may be smaller than the $4.55 \mathrm{~km} \mathrm{~s}^{-1}$ predicted from the ThAr exposures. 
STIS Data We make use of archival STIS observations taken with the E140H and E230H gratings using the $0.20^{\prime \prime} \times 0.09^{\prime \prime}$ apertures. These data were acquired under program 9383 (PI: K. Gordon) and have been previously reported in the literature ${ }^{29}$ The resolution of these data is $R \approx 114,000$ corresponding to $\Delta v \approx 2.75 \mathrm{~km} \mathrm{~s}^{-1}$. The data were processed with CALSTIS v2.27 and otherwise reduced following the discussion in earlier papers. ${ }^{30}$ The $S N R$ of these data are limited by the relatively low UV flux of this highly extincted star for high resolution spectroscopy and ranges between 5 and 15 (per pixel) for the lines of interest.

\section{Hydrogen column density along the Sk 143 sight line}

We derive the $\mathrm{H}$ I column density toward Sk 143 by fitting the strong Ly $\alpha$ absorption seen in the Hubble Space Telescope (HST)/STIS E140H observations. Following Lehner et al., ${ }^{31}$ we fitted Ly $\alpha$ with two components (Galactic and SMC) at fixed velocities derived from the metal absorption lines and the H I 21-cm emission profile for the Galactic component (see below), which is in agreement with the velocity from the absorption profiles. Figure S1] shows the STIS data for the region surrounding $\operatorname{Ly} \alpha$ (binned by 10 pixels for display purposes) along with our best fit $\mathrm{H} \mathrm{I}$ profile and continuum. For the SMC component we find $\log N(\mathrm{H} \mathrm{I})=21.07 \pm 0.05$. We tested the robustness of our fit by undertaking several simulations wherein the velocity centroids of the gas were changed by $\pm 5 \mathrm{~km} \mathrm{~s}^{-1}$, the placement of the continuum was varied, and the continuum was modeled by a range of polynomials of degree of 2 to 4 . The range of values derived in these simulations is used as an estimate of the errors associated with these effects and is included in our final uncertainty. The column density of the Galactic component in the fit is quite uncertain because it is much weaker (see 21-cm emission spectrum discussed below and in Figure $\mathbf{S 2}$ ). Its weakness is in fact a benefit for deriving an accurate SMC $N(\mathrm{H}$ I) as the damping wings are dominated by the SMC component.

Table S1 summarizes previous determinations of the H I column density in the SMC component toward Sk 143. Previous estimates relied on H I 21-cm emission data or Ly $\alpha$ absorption from a low resolution and low signal-to-noise spectrum from the International Ultraviolet Explorer

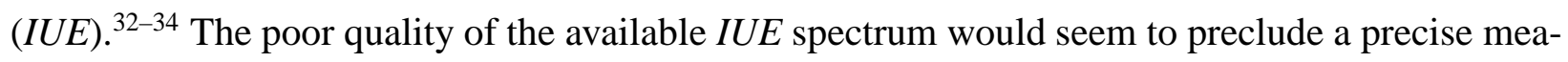




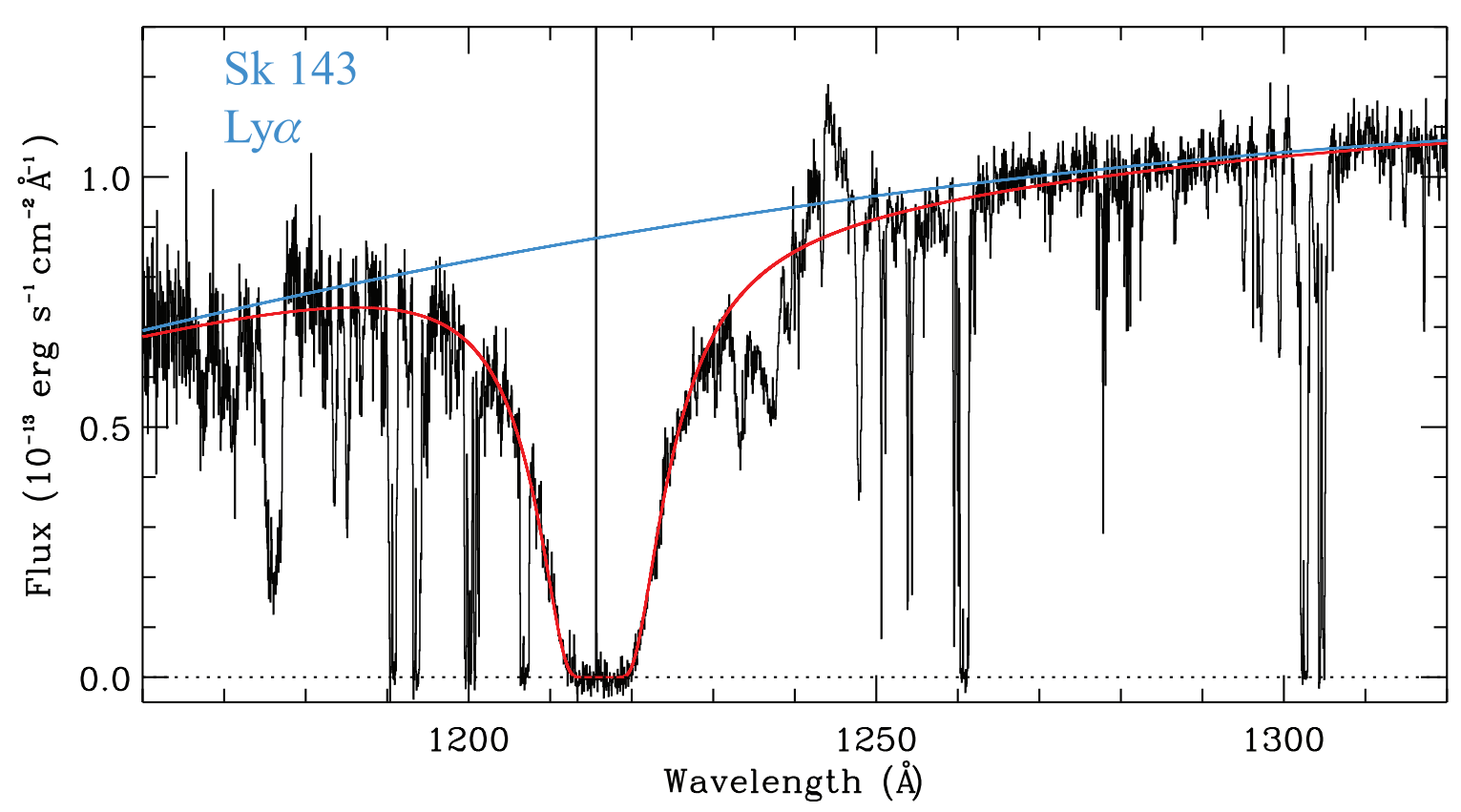

Figure S1: Fit to interstellar H I Ly $\alpha$ absorption observed by HST/STIS against the stellar continuum of Sk 143. The blue line shows the adopted continuum, while the red line shows the continuum absorbed by an $\mathrm{H}$ I column density of $\log N(\mathrm{H} \mathrm{I})=21.07 \pm 0.05$.

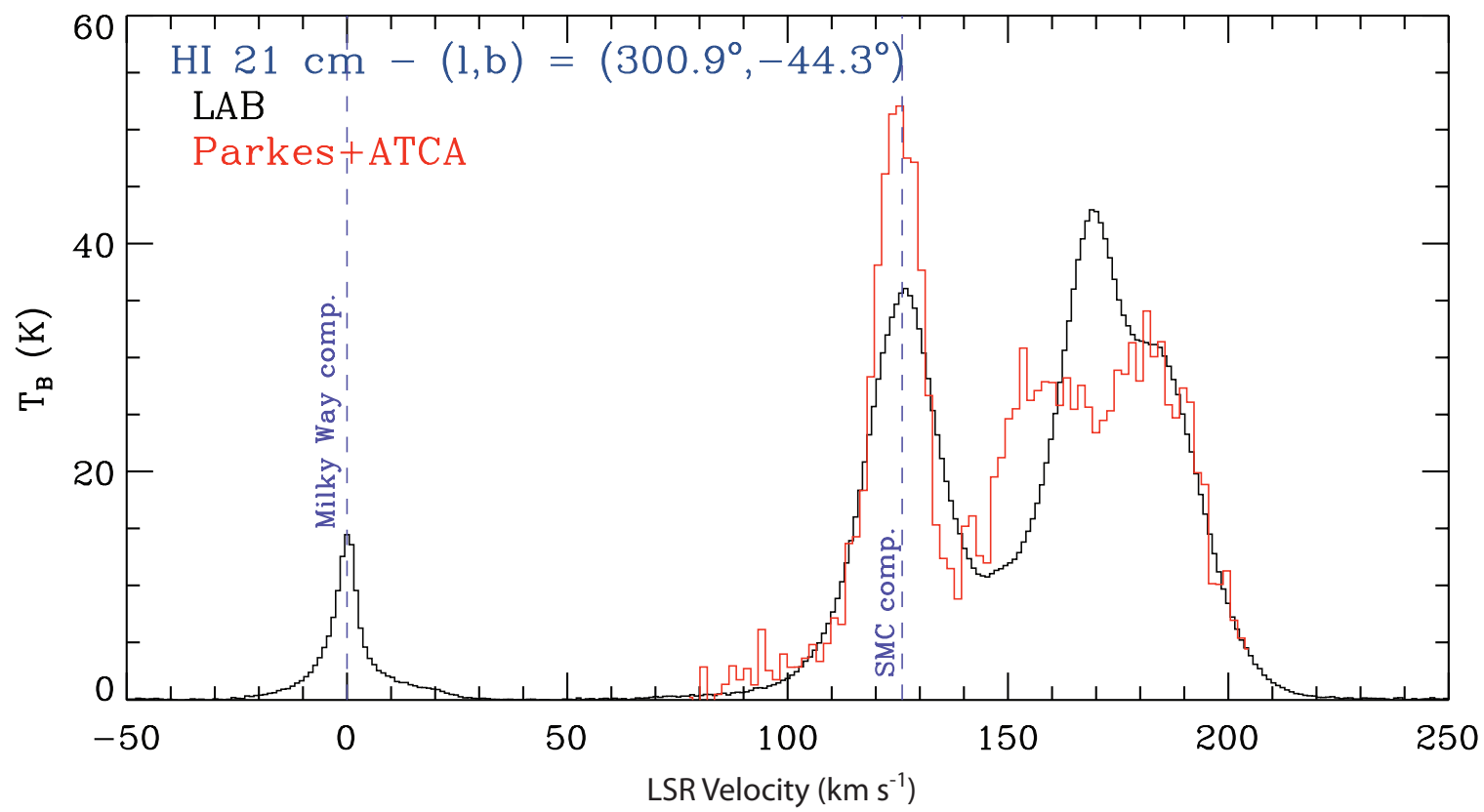

Figure S2: H I 21-cm emission line observations of the sight line to Sk 143. The emission line data in black are taken from the Leiden/Argentine/Bonn (LAB) Survey ${ }^{72}$, with a $36^{\prime}$ beam, while the data in red are taken from the Parkes/ATCA survey of the SMC ${ }^{35]}$, with a $1.6^{\prime}$ beam. The principal SMC component as seen in absorption is marked. 
surement, and the first estimate using those data had a large uncertainty of $40-50 \% .33 \sqrt[34]{3}$ Most recent studies of this sight line $e^{13, \sqrt[143]{32}}$ have adopted $\mathrm{H}$ I estimates derived from the IUE-observed Ly $\alpha$ absorption ${ }^{32 \sqrt{33}}$, or analyses of the H I 21-cm emission line observations..$^{356}$ The most recent determinations using $I U E^{[22}$ give high values of $\log N(\mathrm{H} \mathrm{I}) \approx 21.6$, consistent with values derived by integrating the full SMC profile of the H I 21-cm emission seen in Figure $\mathrm{S} 2$, For comparison with absorption line data toward Sk 143, however, the H I 21-cm emission estimate is not always ideal, as it includes contributions from gas behind the star. Most of the absorption toward Sk 143 is found at $v_{\mathrm{LSR}} \sim 121 \mathrm{~km} \mathrm{~s}^{-1}$ as seen in Figures $\mathbf{S 3}$ and $\mathbf{S 4}$, This component is seen as well in $21-\mathrm{cm}$ emission, but the $21-\mathrm{cm}$ profile contains significant amounts of gas to much larger velocities. Some of this is seen in the absorption line observations, but with a smaller contribution to the total column of undepleted elements (e.g., see the total gas column traced by S II absorption). Integrating the combined Parkes and ATCA H I profile $\mathrm{S}^{3536}$ in Figure $\mathrm{S2}$ over the velocity range containing the majority of the optical/UV metal absorption, $v_{\mathrm{LSR}}=80$ to $146 \mathrm{~km} \mathrm{~s}^{-1}$, we find $\log N(\mathrm{H} \mathrm{I})=21.17 \pm 0.11$ (where the error is a combination of statistical and "beam" errors). Thus, the integration over this smaller velocity range gives results consistent with our measurement and another recent one of Welty \& Crowther ${ }^{37}$ at $\lesssim 2 \sigma$. We note, though, that even though there is a kinematic correspondence in the component at $121 \mathrm{~km} \mathrm{~s}^{-1}$ in the absorption and emission profiles, there is a possible (unknown) uncertainty owing to the unknown depth of the star in the SMC. The moderate difference between our result and that of Welty \& Crowther is due to the difference in the H I column assigned to the Milky Way, for which they derive values from 21-cm measurements while we fit the Ly $\alpha$ directly.

In what follows, we adopt our H I column density measurement from the Ly $\alpha$ fitting. With the $\mathrm{H}_{2}$ column derived from FUSE data, $\frac{15}{15}$ the total hydrogen column along this sight line is then $\log N(\mathrm{H}) \equiv \log \left[N(\mathrm{H} \mathrm{I})+2 N\left(\mathrm{H}_{2}\right)\right]=21.46 \pm 0.04$. The sight line has a high molecular fraction, with $f\left(\mathrm{H}_{2}\right) \equiv 2 N\left(\mathrm{H}_{2}\right) / N(\mathrm{H})=0.6$. 


\section{Table S1: H I column density estimates to Sk 143 using Ly $\alpha$}

\begin{tabular}{lll}
\hline \hline Reference & $\log N(\mathrm{HI})_{\mathrm{SMC}}$ & Data \\
\hline This Work & $21.07 \pm 0.05$ & HST/STIS \\
Welty \& Crowther (2010) $\sqrt{37}$ & 20.95 & HST/STIS \\
Gordon et al. (2003) & $21.60 \pm 0.05$ & IUE \\
Bouchet et al. (1985) & $21.40 \pm 0.17$ & IUE \\
Fitzpatrick (1985) & $21.18 \pm 0.18$ & IUE \\
\hline
\end{tabular}

\section{Metal column densities along the Sk 143 sight line}

Table $\mathrm{S} 2$ gives our adopted final column density for all of the atomic and ionic species detected toward Sk 143 along with the methodology used to derive the columns. We measure metal atom and ion column densities along the Sk 143 sight line using a combination of profile fitting and apparent optical depth integration methods, depending on the quality of the data and the specifics of the individual absorption lines. We assume oscillator strengths from the compilation of Morton ${ }^{\sqrt[38]{ }}$ For each atomic or ionic species, two column densities are given: the first applies to the column density integrated over the entirety of SMC absorption, the second gives the column density associated with the $+121 \mathrm{~km} \mathrm{~s}^{-1}$ component within which the Li I and other neutral species arise. In some cases (e.g., Li I) these are the same as no significant absorption exists outside of this cold, dense component. We use the column densities for this principal component at $v_{\mathrm{LSR}} \approx+121 \mathrm{~km} \mathrm{~s}^{-1}$ in all calculations in the main text, since the Li I-bearing gas is all contained in this component.

Our component fitting column densities are derived using the software package VPFIT ${ }^{39}$ made available by R. Carswell (http://www.ast.cam.ac.uk/ rfc/vpfit.html). VPFIT minimizes the $\chi^{2}$ goodness of fit parameter using a Gauss-Newton type system for parameter updates. $\stackrel{40}{ }$ The free parameters of the fit for each assumed interstellar "component" are the central velocity, Doppler parameter ( $b$-value), and column density of the component. We fit each species independently, with no assumptions about $b$-values or central velocities from other absorbing species (as opposed to assuming the $b$-values are linked through a common temperature and non-thermal velocity). This 
Table S2: Column densities toward Sk 143

\begin{tabular}{lccccc}
\hline \hline Species & $\log N(S M C)$ & $\log N\left(+121 \mathrm{~km} \mathrm{~s}^{-1}\right)$ & Method & $A(\mathrm{X})_{\odot}$ & {$[\mathrm{X} / \mathrm{H}]_{\mathrm{SMC}}$} \\
\hline $\mathrm{H}$ I & $21.07 \pm 0.05$ & $21.07 \pm 0.05$ & 1 & $\equiv 12.00$ & $\ldots$ \\
$\mathrm{H}_{2}$ & $20.93 \pm 0.09$ & $20.93 \pm 0.09$ & 2 & $\equiv 12.00$ & $\ldots$ \\
$\mathrm{H}_{\text {total }}$ & $21.46 \pm 0.04$ & $21.46 \pm 0.04$ & $\ldots$ & $\equiv 12.00$ & $\ldots$ \\
${ }^{7} \mathrm{Li} \mathrm{I}$ & $10.29 \pm 0.02$ & $10.29 \pm 0.02$ & 3 & $3.23 \pm 0.05$ & $-0.55 \pm 0.16$ \\
${ }^{6} \mathrm{Li} \mathrm{I}$ & $9.41 \pm 0.15$ & $9.41 \pm 0.15$ & 3 & $2.14 \pm 0.05$ & $-0.38 \pm 0.21$ \\
$\mathrm{Na}$ I & $12.96 \pm 0.04$ & $12.96 \pm 0.04$ & 3 & $6.26 \pm 0.02$ & $>-2.76 \pm 0.06$ \\
$\mathrm{Mg}$ I & $>13.11 \pm 0.06$ & $>13.11 \pm 0.06$ & 4 & $7.53 \pm 0.01$ & $\ldots$ \\
$\mathrm{Mg}$ II & $15.37 \pm 0.09$ & $15.37 \pm 0.09$ & 3 & $7.53 \pm 0.01$ & $-1.62 \pm 0.10$ \\
S I & $14.15 \pm 0.08$ & $14.15 \pm 0.08$ & 3 & $7.14 \pm 0.02$ & $\ldots$ \\
S II & $\gtrsim 15.36 \pm 0.06$ & $\gtrsim 15.17 \pm 0.05$ & 3 & $7.14 \pm 0.02$ & $\gtrsim-1.24 \pm 0.07$ \\
K I & $12.61 \pm 0.02$ & $12.61 \pm 0.02$ & 3 & $5.08 \pm 0.02$ & $>-1.93 \pm 0.04$ \\
Ca I & $9.42 \pm 0.10$ & $9.42 \pm 0.10$ & 4 & $6.30 \pm 0.02$ & $\ldots$ \\
Ca II & $12.42 \pm 0.01$ & $11.68 \pm 0.01$ & 3 & $6.30 \pm 0.02$ & $>-3.34 \pm 0.04$ \\
Fe I & $11.83 \pm 0.05$ & $11.83 \pm 0.05$ & 4 & $7.45 \pm 0.01$ & $\ldots$ \\
Fe II & $14.63 \pm 0.07$ & $14.15 \pm 0.10$ & 4 & $7.45 \pm 0.01$ & $-2.28 \pm 0.08$ \\
Ti II & $11.88 \pm 0.03$ & $11.47 \pm 0.03$ & 4 & $4.92 \pm 0.03$ & $-2.50 \pm 0.05$ \\
Ni II & $13.10_{-0.11}^{+0.09}$ & $12.97_{-0.10}^{+0.08}$ & 4 & $6.20 \pm 0.01$ & $-2.56 \pm 0.11$ \\
Zn II & $\gtrsim 12.80 \pm 0.06$ & $\gtrsim 12.80 \pm 0.06$ & 4 & $4.60 \pm 0.03$ & $\gtrsim-1.26 \pm 0.07$ \\
\hline
\end{tabular}

Methods used for determining column densities: (1): Ly $\alpha$ Fitting; (2): Fitting by Cartledge et al. ${ }^{[15}(3)$ : Profile fitting; (4): $N_{a}(v)$ integration. The values of $[\mathrm{X} / \mathrm{H}]_{\mathrm{SMC}}$ are gas-phase abundances, i.e., they reflect the sub-solar SMC metallicity and the differential incorporation of elements into the solid phase (dust grains). 
has the advantage that additional unsuspected components should not adversely affect the results, so long as the lines are not strongly saturated. We fit multiplets simultaneously where available. We did not, however, simultaneously fit the strongest doublets of $\mathrm{Na} \mathrm{I}$ and $\mathrm{K}$ I with the weakest doublets available (see Figures $\underline{\mathrm{S} 3}$ and $\mathrm{S} 4$ for the observed lines). The column densities in Table S2 are derived based on the weaker doublets of these species due to the extreme saturation of the strongest lines.

Our fitting of all of the neutral species suggests that the SMC absorption seen in weak lines of neutral species (i.e., not including the Na I D lines) are well described by a single component. We see no compelling evidence for additional components that contribute significantly to the column densities of the neutral species. The high resolution CES data presented by Welty et al. ${ }^{14}$ are consistent with a single dominant component with only very weak additional components at higher relative velocities. We note that the presence of additional unknown components could affect the interpretation of the $\mathrm{Li}$ isotopic ratio if they had strong variations in the ratio between components or if there were moderate strength components present with separations matching the isotope shift. For derivation of total column densities of ${ }^{7} \mathrm{Li}$ (and largely even ${ }^{6} \mathrm{Li}$ ), the presence of multiple components is not particularly important. In general our profile fits to these neutral species gave $b$-values of order $0.7 \mathrm{~km} \mathrm{~s}^{-1}$, consistent with previous studies of this sight line ${ }^{13}$ We note also that the presence of additional unidentified components is unlikely unless they have a separation less than this $0.7 \mathrm{~km} \mathrm{~s}^{-1}$.

Our derived column densities of $\mathrm{Na} \mathrm{I,} \mathrm{K} \mathrm{I,} \mathrm{and} \mathrm{Ca} \mathrm{II} \mathrm{vary} \mathrm{somewhat} \mathrm{from} \mathrm{these} \mathrm{earlier}$ works $^{1314}$ (with our columns being higher) because we have generally studied weaker lines, fully fit multiple lines, or in the case of Ca II used a careful treatment of the continuum for the weak line, which lies on the stellar $\mathrm{H} \epsilon$ absorption requiring special techniques for the continuum assessment.

Our fitting assumed Gaussian LSFs with breadths derived as described above for the UVES observations of Li I. For the blue CCD data we had no higher resolution comparison observations, so we adopted the LSF breadth suggested by the calibration lamp data. There was no obvious indication that this LSF is too broad. For fitting the STIS E140H observations of Mg II, we 


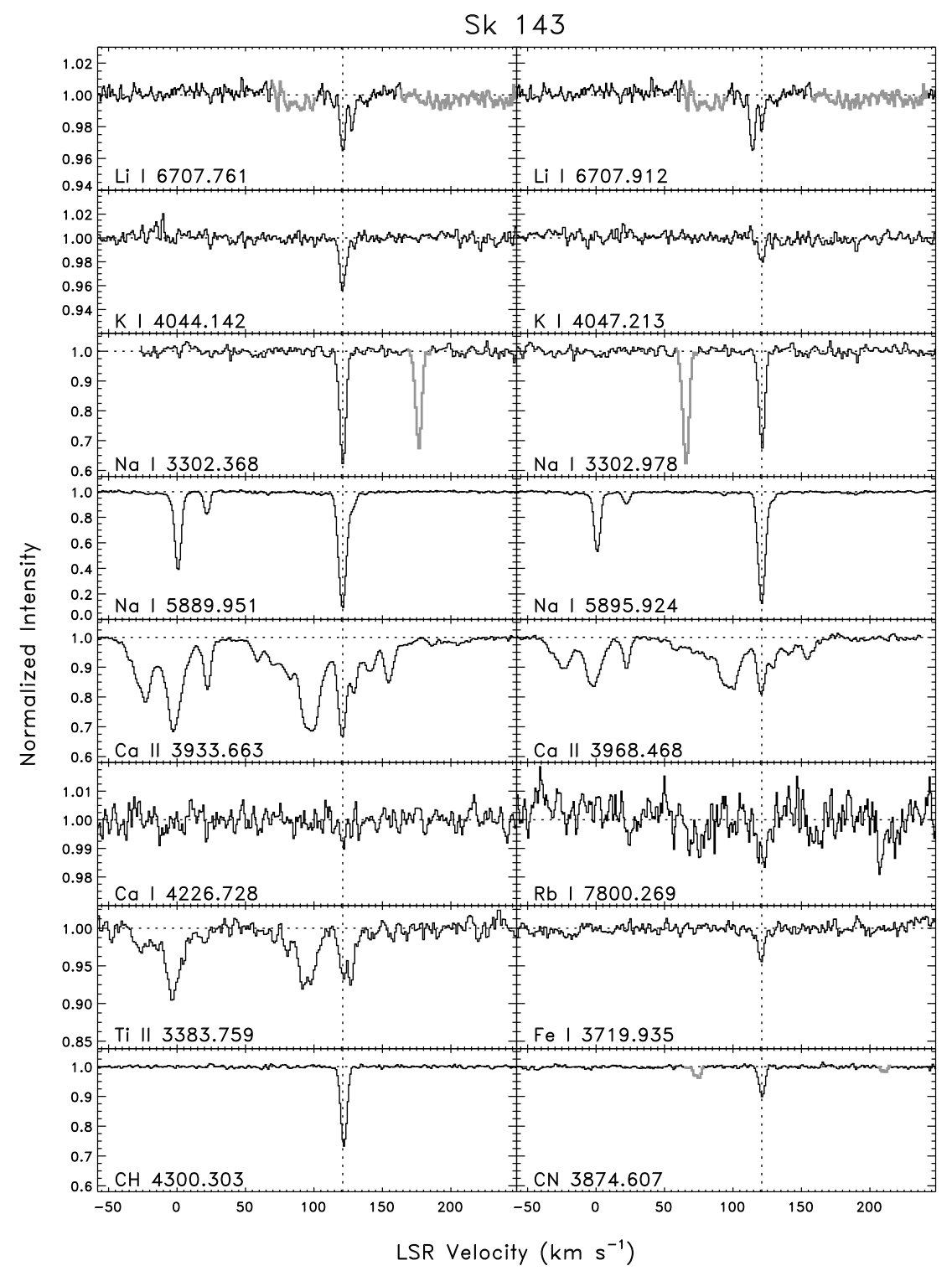

Figure S3: Normalized interstellar absorption profiles for optical metal line transitions toward Sk 143 plotted versus LSR velocity. These data are from the VLT+UVES observations of Sk 143. Contaminating absorption from other species are marked in light gray. 


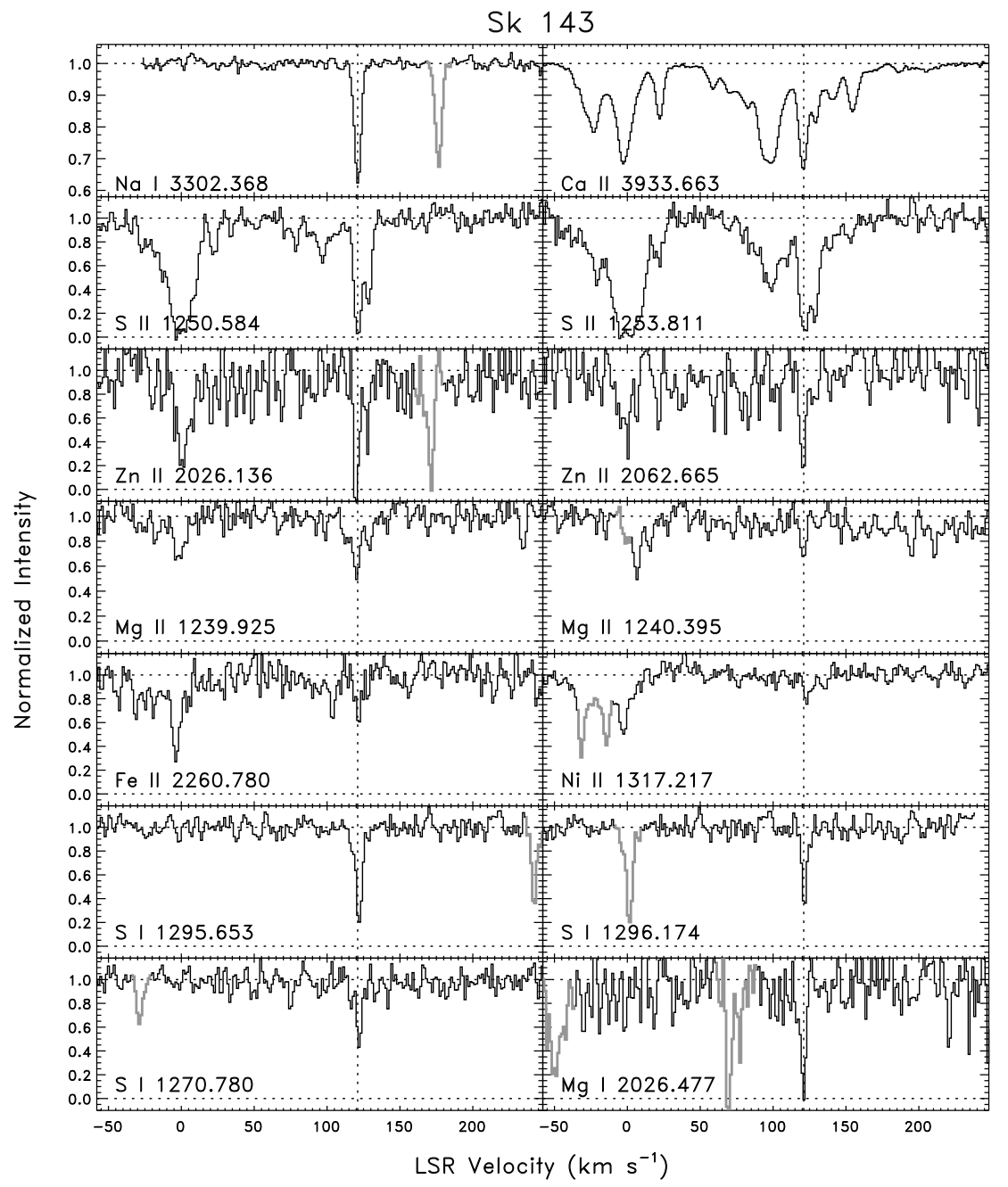

Figure S4: Normalized interstellar absorption profiles for ultraviolet metal line transitions toward Sk 143 plotted versus LSR velocity. These data are from the HST/STIS E140H and E230H observations of Sk 143. Contaminating absorption from other species are marked in light gray. Optical observations from the previous figure are shown at the top for comparison. 
assumed a breadth of $2.75 \mathrm{~km} \mathrm{~s}^{-1}$ (FWHM). The STIS LSF is not strictly a Gaussian. In our case we were only interested in separating the SMC Mg II absorption from the contaminating Milky Way absorption. For these purposes and with the strength of the lines, the assumption of a Gaussian LSF will not adversely affect the results.

We have used profile fitting to derive column densities for neutral species with small $b$ values in case unresolved saturation is present or where blending is a concern. For most of the ionic species we adopt column densities derived through an integration of the apparent column density, $N_{a}(v)$, profiles, ${ }^{41}$ largely following methodologies in our earlier works ${ }^{42}$ Saturation can be identified by comparing the $N_{a}(v)$ profiles from two lines of an absorbing species with significantly different oscillator strengths ( $f$-values). The entries in Table $\mathbf{S 2}$ listed as lower limits are those for which we have evidence for unresolved saturation.

Table $\left[2\right.$ also gives the solar system abundances ${ }^{23}$ for each element and the relative gasphase abundance of the element in the ISM of the SMC, $[\mathrm{X} / \mathrm{H}]_{\mathrm{SMC}}$. The latter quantity reflects the base subsolar metallicity of the present-day $\mathrm{SMC},[\mathrm{Fe} / \mathrm{H}]_{\mathrm{SMC}}=-0.59 \pm 0.06$ derived from a straight mean of observations of early type stars,,$\sqrt[43]{46}$ and the effects of differential dust depletion of the elements. ${ }^{47}$ Where two ionization states are measured, we only give the gas-phase values for the most abundant ion. Inequalities either reflect the non-dominant nature of the species (e.g., for the neutrals) or the probable presence of unresolved saturation. The abundances listed for the $\mathrm{Li}$ isotopes are drawn from the main text (i.e., adopting the abundances derived from scaling the Li I/K I ratio).

Assessing the effects of dust depletion on the gas along this sight line is somewhat complicated by the lack of a quality measurement of an undepleted metal species. The $[\mathrm{X} / \mathrm{H}]$ values in Table $\$ 2$ measure the effects of the sub-solar metallicity of the SMC and its non-solar relative abundance pattern in addition to dust depletion effects. Welty \& Crowther ${ }^{\sqrt{37}}$ have recently estimated the intrinsic Ti abundance of the SMC based on early-type stars, deriving an estimate for the present day abundance of $A(\mathrm{Ti})_{\mathrm{SMC}} \approx 4.28$. Our measurements of the gas-phase Ti II then suggest Ti is depleted by -1.86 dex relative to the intrinsic SMC abundance; sight lines in the Milky Way have 
depletions -2 to -3 for similar $E(B-V)$ or $\mathrm{CN}$ columns, but it is not dissimilar from sight lines in the Galactic disk with modest depletions. ${ }^{[37}$ Defining the reference abundance can be difficult for assessing the depletion of an element. Another approach to understanding the level of elemental incorporation into grains is to measure the differential depletion between two metals. The least de-

pleted species with a good measure of the total column density in the table is that of $\mathrm{Mg}$, for which we have a good measure of the dominant ion $\mathrm{Mg}$ II. Comparing strongly-depleted metals to $\mathrm{Mg}$ in the SMC gas toward Sk 143 gives $[\mathrm{Fe} / \mathrm{Mg}]_{\mathrm{SMC}}=-0.66 \pm 0.13,[\mathrm{Ni} / \mathrm{Mg}]_{\mathrm{SMC}}=-0.94 \pm 0.15$, and $[\mathrm{Ti} / \mathrm{Mg}]_{\mathrm{SMC}}=-0.88 \pm 0.11$. The uncertainties are large given the low SNR of the STIS data for deriving abundances, so comparing these data to Milky Way sight lines does not lead to great insight. These are consistent with relative gas-phase abundances seen in Milky Way disk gas for sight lines with low to modest depletion (at least for the disk). ${ }^{47,49}$ Measurements of Li in the Milky Way typically probe sight lines or clouds with stronger depletions than these values; the Li depletion effects should not as strong in this SMC gas as in the gas typically probed in the Milky Way.

\section{Lithium abundance estimates}

Several estimates for the gas-phase ${ }^{7} \mathrm{Li}$ abundance are given in the text, which are summarized in Table $\mathrm{S3}$, Estimating the gas-phase ${ }^{7} \mathrm{Li}$ abundance from measurements of $N\left({ }^{7} \mathrm{Li} \mathrm{I}\right) / N(\mathrm{H})$ requires the application of an ionization correction to account for the unseen ${ }^{7} \mathrm{Li}$, which is mostly in its singly ionized form in the ISM. Assuming ionization rate balance and only atomic processes, $N(\mathrm{Li}) / N(\mathrm{Li} \mathrm{I}) \approx N(\mathrm{Li}$ II $) / N(\mathrm{Li} \mathrm{I})=\Gamma(\mathrm{Li} \mathrm{I})\left[n_{e} \alpha(\mathrm{Li} \mathrm{II}, T)\right]^{-1}$, where $\Gamma(\mathrm{Li}$ I $)$ is the photoionization rate and $\alpha(\mathrm{Li}$ II, $T)$ the recombination coefficient. (We generically refer to $\mathrm{Li}$ I here, which applies to either isotope or their sum.) At first glance it would seem that knowledge of $\Gamma$ and $n_{e}$ are crucial to estimating the total abundance. However, similar equations can be written for observed adjacent ionization states, notably for $N(\mathrm{Ca}$ II $) / N(\mathrm{Ca} \mathrm{I})$ and $N(\mathrm{Fe}$ II $) / N(\mathrm{Fe} \mathrm{I})$. Substituting $n_{e}$ derived from these ratios into the expression for Li gives, for example,

$$
\frac{N(\mathrm{Li} I \mathrm{II})}{N(\mathrm{Li} \mathrm{I})}=\frac{N(\mathrm{Fe} \text { II })}{N(\mathrm{Fe} \mathrm{I})} \frac{\Gamma(\mathrm{Li} \mathrm{I}) / \Gamma(\mathrm{Fe} \mathrm{I})}{\alpha(\mathrm{Li} \text { II }, T) / \alpha(\mathrm{Fe} I I, T)}
$$




\section{Table S3: Summary of SMC lithium abundances}

\begin{tabular}{lcl}
\hline \hline Quantity & Value & Methodology \\
\hline$A\left({ }^{7} \mathrm{Li}\right)_{\mathrm{SMC}}$ & $2.68 \pm 0.16$ & Scaled from ${ }^{7} \mathrm{Li} \mathrm{I} / \mathrm{K} \mathrm{I}$ \\
$A\left({ }^{7} \mathrm{Li}\right)_{\mathrm{SMC}}$ & $2.38 \pm 0.17$ & Scaled from ${ }^{7} \mathrm{Li} \mathrm{I} / \mathrm{S} \mathrm{I}$ \\
$A\left({ }^{7} \mathrm{Li}\right)_{\mathrm{SMC}}$ & $3.01 \pm 0.12$ & Ionization correction from Ca II/Ca I \\
$A\left({ }^{7} \mathrm{Li}\right)_{\mathrm{SMC}}$ & $2.79 \pm 0.11$ & Ionization correction from Fe II/Fe I \\
{$\left[{ }^{7} \mathrm{Li} / \mathrm{K}\right]_{\mathrm{SMC}}$} & $+0.04 \pm 0.10$ & Differential ionization correction \\
{$\left[{ }^{7} \mathrm{Li} / \mathrm{S}\right]_{\mathrm{SMC}}$} & $-0.26 \pm 0.11$ & Differential ionization correction \\
\hline
\end{tabular}

All absolute and relative abundances of SMC Li derived using several different methods are given. The recommended values are those derived by comparison with $\mathrm{K}$, i.e., $A\left({ }^{7} \mathrm{Li}\right)_{\mathrm{SMC}}=2.68 \pm 0.16$ and $\left[{ }^{7} \mathrm{Li} / \mathrm{K}\right]_{\mathrm{SMC}}=+0.04 \pm 0.10$. The former relies on the application of several scale factors, including a differential ionization correction, the metallicity of the SMC, and an estimated $\mathrm{K} / \mathrm{Fe}$ abundance. The latter relies on a differential ionization correction, but one that is well founded in the observed Galactic relationship between Li I and K I.

While the individual photoionization rates depend on the intensity of the interstellar radiation field, to first order the ratio of photoionization rates $\Gamma(\mathrm{Li} \mathrm{I}) / \Gamma(\mathrm{Fe} \mathrm{I})$ does not. What is crucial in this case is the shape of the radiation field over the range of energies encompassed by the ionization edges of these neutrals. The grossly similar temperature sensitivities for the recombination coefficients mitigates the effect of this unknown, as well. The total abundance of $\mathrm{Li}$ is then $\log \left[N\left({ }^{7} \mathrm{Li}\right) / N(\mathrm{H})\right]=\log \left[N\left({ }^{7} \mathrm{Li} \mathrm{I}\right) / N(\mathrm{H})\right]-\log x(\mathrm{Li} \mathrm{I})$, where the term $-\log x(\mathrm{Li} \mathrm{I})$ is the ionization correction factor and $x(\mathrm{Li} \mathrm{I}) \equiv N(\mathrm{Li} \mathrm{I}) / N(\mathrm{Li})$ is the ionization fraction of $\mathrm{Li}$ in neutral form.

As discussed in the main text, we derive ionization corrections for the $\mathrm{Li} / \mathrm{H}$ comparison on the basis of the observed ratios $N(\mathrm{Ca}$ II $) / N(\mathrm{Ca} \mathrm{I})$ and $N(\mathrm{Fe} \mathrm{II}) / N(\mathrm{Fe} \mathrm{I})$. These give ionization corrections of $-\log x(\mathrm{Li} \mathrm{I})=+1.96 \pm 0.10$ and $+2.18 \pm 0.12$ from the $\mathrm{Ca}$ and Fe ions, respectively, assuming standard relative ionization rates with specific interstellar radiation fields.$^{20 \mid[5]}$ These are derived using column densities from the $v_{\mathrm{LSR}} \approx+121 \mathrm{~km} \mathrm{~s}^{-1}$ cloud only. The ratio of ${ }^{7} \mathrm{Li}$ I to 
total hydrogen in the SMC gas along this sight line is $\log \left[N\left({ }^{7} \mathrm{Li} \mathrm{I}\right) / N(\mathrm{H})\right]=-11.17 \pm 0.04$, giving $\log \left[N\left({ }^{7} \mathrm{Li}\right) / N(\mathrm{H})\right]=-9.21 \pm 0.11$ and $-8.99 \pm 0.13$. While we also have data covering adjacent ions of $\mathrm{S}$ and $\mathrm{Mg}$, we have only limits for one ionization state of each of these elements that do not produce useful limits on the ionization corrections.

The discrepancies between these calculations are in part due to errors in the calculated photoionization rates and recombination coefficients. However, it is also likely that the neutral and singly-ionized $\mathrm{Ca}$ and $\mathrm{Fe}$, which have different ionization potentials, photoionization cross sections, and recombination coefficients, trace slightly different conditions within the cloud. The conditions traced by these species will also be different than those traced by Li I and Li II, limiting the precision of this approach. In fact, electron densities derived from various tracers can vary, with Ca I typically predicting significantly larger $n_{e}$ than most. ${ }^{20}$ In the SMC cloud probed here, however, the Ca I column seems to be unusually low, as indicated by the Ca I to K I ratio, for example, compared with Milky Way sight lines. The integrated $N(\mathrm{Ca}$ II $) / N(\mathrm{Ca}$ I $)$ is higher than all measured values in the compilation of Welty et al.20 (although some sight lines with nondetections of $\mathrm{Ca}$ I could have higher values), although the ratio in the $v_{\mathrm{LSR}} \approx+121 \mathrm{~km} \mathrm{~s}^{-1}$ component is near the mean of those measurements.

Using $\mathrm{Li} \mathrm{I} / \mathrm{K} \mathrm{I}$ as a tracer of the total interstellar $\mathrm{Li} / \mathrm{K}$ abundance follows a similar approach:

$$
\frac{N(\mathrm{Li} I \mathrm{II})}{N(\mathrm{~K} \mathrm{II})}=\frac{N(\mathrm{Li} \mathrm{I})}{N(\mathrm{~K} \mathrm{I})} \frac{\Gamma(\mathrm{Li} \mathrm{I}) / \Gamma(\mathrm{K} \mathrm{I})}{\alpha(\mathrm{Li} I I, T) / \alpha(\mathrm{K} \mathrm{II}, T)} .
$$

In this case the ratio of relative ionization rates to recombination coefficients gives an ionization correction of $+0.54 \pm 0.08$ (following Steigman ${ }^{19}$ in adopting an error based on the range of recombination coefficients derived from a wide range of temperatures). The $\mathrm{Li} \mathrm{I} / \mathrm{K}$ I ratio is powerful given the very nearly linear relationship between the column densities of these species in the Milky Way ${ }^{1822}$ We assume the same physics applies in the SMC, and thus this ratio should be similarly useful there. This implies Li and $\mathrm{K}$ have very similar dust depletion ${ }^{18} \mid 51$ and ionization conditions that track each other well. Similarly, the K I/S I ratio is a power law with an index somewhat greater than unity ${ }^{[20}$ The tight correlation with larger power law slope likely implies the two neutrals trace similar physical conditions, but with different depletion characteristics. We make the 
assumption that Li I also traces physical conditions similar to those of S I-bearing clouds. The ionization correction relating $\mathrm{Li} \mathrm{I} / \mathrm{S} \mathrm{I}$ to $\mathrm{Li} / \mathrm{S}$ is $-0.32 \pm 0.08 \mathrm{dex}$. We do not use the other neutral tracers for such a derivation of the Li to metal nuclei ratio because of the weaker correlations and evidence for differeng physical conditions between Li I and the remaining neutrals.

Transforming $\left[{ }^{7} \mathrm{Li} / \mathrm{K}\right]_{\mathrm{SMC}}$ to an estimate of $A\left({ }^{7} \mathrm{Li}\right)_{\mathrm{SMC}}$ involves a number of scale factors. Our adopted mean metallicity of the SMC $\left([\mathrm{Fe} / \mathrm{H}]_{\mathrm{SMC}}\right)$ is described above. One other scale factor is the abundance of $\mathrm{K} / \mathrm{Fe}$ with respect to the solar system. The $\mathrm{K} / \mathrm{Fe}$ ratio is not measured directly in the SMC. However, $\mathrm{K}$ has its origins in explosive oxygen burning in high mass stars and tends to behave nucleosynthetically like an $\alpha$ element. ${ }^{52}$ Studies of early-type stars, ${ }^{45[53 \sqrt[54]{54}} \mathrm{A}$ supergiants, $\underset{55}{ }$ and $\mathrm{H}$ II regions ${ }^{56}$ suggest $[\mathrm{S}, \mathrm{Si} / \mathrm{Fe}] \approx 0$ in the present-day SMC. These studies can have significant uncertainties, and there is some dispersion in the results. We adopt a value $[\mathrm{K} / \mathrm{Fe}]_{\mathrm{SMC}}=+0.0 \pm 0.1$ in our analysis (uncertainty from dispersion in the $\alpha$ to Fe dispersion in the SMC). We note that this is at odds with what is seen in the Milky Way. The mean of two samples of Milky Way stars within \pm 0.1 dex of the SMC metallicity, $\left[5758[\mathrm{~K} / \mathrm{Fe}]_{\mathrm{SMC}}=+0.27 \pm 0.09\right.$ (standard deviation). The discrepancy is due to the significantly different star formation and enrichment history of the SMC compared with the Milky Way. $\underline{[5]}$

Our ${ }^{7} \mathrm{Li}$ abundance and ${ }^{7} \mathrm{Li} / \mathrm{K}$ ratio is significantly higher than that implied ${ }^{19}$ by the upper limits $^{\sqrt[60]{62}}$ or claimed detections ${ }^{63}$ toward SN1987A in the Large Magellanic Cloud (LMC). With an abundance roughly twice that of the SMC, the LMC should have a ${ }^{7} \mathrm{Li}$ abundance at least as high as that of the SMC. The values reported for this sight line vary significantly, and we view them with significant skepticism in hindsight. Aside from the discrepancies in the Li I measurements, there are worries about saturation corrections for the K I reference. Furthermore, the sight line to SN1987A probes a significantly lower column density, more highly ionized portion of the ISM. This sight line does not favor the formation of atomic species such as Li I, making the ionization effects even more severe. Future observations of Li I absorption within the LMC should provide more clarity to the chemical evolution of $\mathrm{Li}$ in the Clouds. 


\section{Lithium Isotopic Ratio}

The $\mathrm{Li}$ isotope ratio can provide some constraints on the production of $\mathrm{Li}$ and any non-standard contributions to $\mathrm{Li}$ since ${ }^{6} \mathrm{Li}$ is not expected to be produced in significant amounts in $\mathrm{BBN}+{ }^{10}$ Our estimate of the ${ }^{6} \mathrm{Li} /{ }^{7} \mathrm{Li}$ relies on simultaneously fitting the hyperfine structure of the ${ }^{7} \mathrm{Li}$ I and ${ }^{6} \mathrm{Li}$ I absorption. We adopt wavelengths for the hyperfine levels from Sansonetti et al ${ }^{64}$ and $f$-values summarized in Welty et al. ${ }^{65}$ We assume the ISM absorption from the neutral species is wellcharacterized by a single component or cloud and that both isotopes have a common $b$-value. The latter assumption is only strictly correct if the velocity dispersion is dominated by non-thermal motions (which is likely), although it has little practical effect given the weakness of the Li I absorption. We fit a stellar continuum to the data before the fitting process. The continuum in the region around Li I was well fit with a first-order Legendre polynomial. We assessed higher order fits using an $F$-test, but they did not provide a statistically significant improvement in the goodnessof-fit parameter over the region used to derive the continuum. In the profile fitting process, we allow the parameters of the continuum fit to vary during the minimization process. This allows VPFIT both to esimate the continuum fit objectively and to include an estimate of the uncertainties caused by continuum fitting into the final error budget.

The column densities reported in Table $\$ 2$ are derived from these fits. The best fit Doppler parameter is $b=0.8 \pm 0.5 \mathrm{~km} \mathrm{~s}^{-1}$. Because the absorption is so weak, the $b$-value has a minor effect on the quality of the column density determination (as would multiple components). The fits were made with the UVES LSF as discussed above. The resolution we derive for the red side data are higher than typically advertised or adopted for the instrument. Our error budget includes a contribution derived by refitting the data with LSF breadths varied by $\pm 1 \sigma$, but the resulting differences in the central value are minimal. Adopting a significantly broader FWHM for the LSF (e.g., $\Delta v \approx 4.45 \mathrm{~km} \mathrm{~s}^{-1}$ as suggested by the ThAr lines) has very little effect on the central value or uncertainties, although the $\chi^{2}$ goodness-of-fit parameter is larger. In this case, the profiles appear visibly broader than the data, with the peaks of the models underpredicting the observed absorption in the core and the wings overpredicting the observed absorption in the edges of the profiles. We have tested the impact of adding additional components, but VPFIT rejects them as 
unnecessary as they do not significantly improve the fit.

It is important to point out that the potential presence of ${ }^{6} \mathrm{Li} \mathrm{I}$ absorption is not simply a function of the manner in which we have proceeded with the profile fitting. The ${ }^{6} \mathrm{Li}$ I contribution is hinted at from simple equivalent width measurements of the ${ }^{7} \mathrm{Li}$ I doublet. Using our initial continuum (i.e., not the final one adopted above through the formal fitting process), the weak and strong lines of ${ }^{7} \mathrm{Li}$ I give equivalent widths $W_{\lambda}=3.77 \pm 0.23 \mathrm{~m} \AA$ and $2.40 \pm 0.22 \mathrm{~m} \AA$. (We caution both the errors and equivalent widths derived here may not be equivalent to those reported through the profile fitting analysis due to the different manner in which the continuum placement uncertainties are included and the crude nature of the separation of the strong and weak lines in the direct integration of equivalent widths.) Assuming there is no saturation, a safe assumption given the weakness of the lines, these lines should have equivalent widths in a 2:1 ratio. The observed ratio of $1.57: 1$ is lower due to the blended contribution of ${ }^{6} \mathrm{Li}$ I to the weak member of the ${ }^{7} \mathrm{Li}$ I doublet. A simple estimate of the ${ }^{6} \mathrm{Li}$ I column density can be derived by subtracting half of the ${ }^{7} \mathrm{Li}$ I strong line equivalent width from the weak line value. This yields $\log N\left({ }^{6} \mathrm{Li} \mathrm{I}\right) \approx 9.41 \pm 0.20$, completely consistent with the value derived from the profile fitting.

The derived isotopic ratio for the SMC gas toward Sk 143 is ${ }^{6} \mathrm{Li} /{ }^{7} \mathrm{Li}_{\mathrm{SMC}}=0.13 \pm 0.05$, which implies a limit ${ }^{6} \mathrm{Li} /{ }^{7} \mathrm{Li}_{\mathrm{SMC}}<0.28(3 \sigma)$. The limit itself does not constrain the ratio in an interesting way. It is, for example, consistent with the Solar System value, $\left({ }^{6} \mathrm{Li} /{ }^{7} \mathrm{Li}\right)_{\odot}=0.0787 \pm$ $0.0004, \underline{66}$ and it is very similar to a number of measurements in Milky Way gas, $\frac{17}{18}$ although there are clouds and sight lines with large variations from this value. ${ }^{67}$ Kawanomoto et al ${ }^{17}$ have recently derived a mean for the Milky Way ISM of $\left\langle{ }^{6} \mathrm{Li} /{ }^{7} \mathrm{Li}\right\rangle_{\mathrm{MW}}=0.13 \pm 0.04$. The only known source of post-Big Bang ${ }^{6} \mathrm{Li}$ is production via cosmic ray interactions with interstellar medium (ISM) particles, either through spallation or $\alpha+\alpha$ fusion $.68+70$ For standard energy distributions of Galactic cosmic rays, these isotopes are produced in a ratio ${ }^{6} \mathrm{Li} /{ }^{7} \mathrm{Li} \approx 0.67$, while non-standard assumptions can push this ratio to 0.5 or a bit lower. ${ }^{71}$ For the standard production ratio, our central value implies that $(19 \pm 8) \%$ of the ${ }^{7} \mathrm{Li}$ in the ISM of the SMC has been produced via cosmic rays, with a limit of $<42 \%(3 \sigma)$. 
Our data show ${ }^{6} \mathrm{Li}$ I at $<3 \sigma$ significance, and the reality of the ${ }^{6} \mathrm{Li}$ I at the levels of our best fit value should rightfully be viewed with caution. Sk 143 is bright enough that follow-up observations should be able to significantly reduce the statistical uncertainties without much difficulty. We have not pushed the limits of UVES with the present observations. At the same time, measurement of $A\left({ }^{6} \mathrm{Li}\right)$ in sub-solar metallicity environments are potentially incredibly important. A significant abundance of ${ }^{6} \mathrm{Li}$ in low metallicity environments could come from pre-galactic cosmic rays accelerated via the collapse of large scale structure (producing Li via $\alpha+\alpha$ fusion) ${ }^{28}$ or even from the effects of non-Standard Model particles in the early universe that affect BBN ${ }^{10}$ Future observations of the gas along this and other SMC or LMC sight lines may be the best way to reliably constrain the Li isotopic ratio at sub-solar metallicities.

\section{Notes on Chemical Evolution Models}

We compare our results in Figures 2 and 3 with those of recent chemical evolution models of Prantzos, ${ }^{25}$ although there are other models in the literature. ${ }^{26,73}$ These models account for postBBN contributions to the total ${ }^{7} \mathrm{Li}$ abundance from massive stars through neutrino nucleosynthesis in core collapse supernova $e^{\frac{76}{6}}$ and from low mass stars as red giant or asymptotic giant branch stars or novae. .25 The ${ }^{7} \mathrm{Li}$ yields from these sources are poorly known. Both ${ }^{7} \mathrm{Li}$ and ${ }^{6} \mathrm{Li}$ are synthesized directly in the ISM through cosmic ray nucleosynthesis.

The Prantzos models have worked around the uncertainties in the stellar yields by assuming the low-mass star contributions are sufficient to reproduce the solar system (meteoritic) abundances. The relative contributions of the various sources as a function of metallicity follow the relative importance of core collapse (high mass) and type Ia (low mass) supernovae ( $\mathrm{SNe}$ ). The cosmic ray model adopted follows from an analysis of the the energy input from core collapse $\mathrm{SNe}$

of massive stars that have suffered mass loss through stellar winds. ${ }^{[25}$ In assessing the yields, no credence is given to the $\mathrm{Li}$ abundances of stars in the Milky Way. The only data points matched by the models are the primordial and meteoritic abundances of $\mathrm{Li}$.

While the uncertainties in the yields are significant, these models are illustrative of the shape of the post-BBN evolution of cosmic Li. However, the primordial and solar system abundances 
adopted by Prantzos are different than those adopted in our work. Thus, for comparison with our measurements, we have had to make adjustments to the model results. We have done this by adopting the Cyburt et al. ${ }^{\frac{3}{3}}$ primordial abundance, assuming the contributions from core-collapse SNe and cosmic rays are as given in Prantzos' work, and independently scaling his adopted lowmass star production rates to match our adopted solar system abundance..$^{23}$

29. Sofia, U.J., Gordon, K.D., Clayton, G.C., Misselt, K., Wolff, M.J., Cox, N.L.J., \& Ehrenfreund, P. Probing the Dust Responsible for Small Magellanic Cloud Extinction. Astrophys. J., 636, 753-764 (2006)

30. Lehner, N., \& Howk, J. C. Highly ionized plasma in the Large Magellanic Cloud: evidence for outflows and a possible galactic wind. Mon. Not. R. Astron. Soc., 377, 687-704 (2007)

31. Lehner, N., Howk, J. C., Keenan, F. P., \& Smoker, J. V. Metallicity and Physical Conditions in the Magellanic Bridge. Astrophys. J., 678, 219-233 (2008)

32. Gordon, K. D., Clayton, G. C., Misselt, K. A., Landolt, A. U., \& Wolff, M. J. A Quantitative Comparison of the Small Magellanic Cloud, Large Magellanic Cloud, and Milky Way Ultraviolet to Near-Infrared Extinction Curves. Astrophys. J., 594, 279-293 (2003)

33. Fitzpatrick, E. L. Ultraviolet interstellar absorption toward stars in the Small Magellanic Cloud. III - The structure and kinematics of the Small Magellanic Cloud. Astrophys. J. Suppl., 59, 77-93 (1985)

34. Bouchet, P., Lequeux, J., Maurice, E., Prevot, L., \& Prevot-Burnichon, M. L. The visible and infrared extinction law and the gas-to-dust ratio in the Small Magellanic Cloud. Astron. \& Astrophys., 149, 330-336 (1985)

35. Stanimirovic, S., Staveley-Smith, L., Dickey, J. M., Sault, R. J., \& Snowden, S. L. The largescale HI structure of the Small Magellanic Cloud. Mon. Not. R. Astron. Soc., 302, 417-436 (1999) 
36. Stanimirović, S., Staveley-Smith, L., \& Jones, P. A. A New Look at the Kinematics of Neutral Hydrogen in the Small Magellanic Cloud. Astrophys. J., 604, 176-186 (2004)

37. Welty, D. E., \& Crowther, P. A. Interstellar TiII in the Milky Way and Magellanic Clouds. Mon. Not. R. Astron. Soc., 404, 1321-1348 (2010)

38. Morton, D. C. Atomic Data for Resonance Absorption Lines. III. Wavelengths Longward of the Lyman Limit for the Elements Hydrogen to Gallium. Astrophys. J. Suppl., 149, 205-238 (2003)

39. Webb, J. K. Quasar Absorption Lines. Ph.D. Thesis, (1987)

40. Murphy, M. T. Probing variations in the fundamental constants with quasar absorption lines. Ph.D. Thesis, (2002)

41. Savage, B. D., \& Sembach, K. R. The analysis of apparent optical depth profiles for interstellar absorption lines. Astrophys. J., 379, 245-259 (1991)

42. Howk, J. C., Sembach, K. R., \& Savage, B. D. Ionized Gas in the First 10 Kiloparsecs of the Interstellar Galactic Halo. Astrophys. J., 586, 249-267 (2003)

43. Trundle, C., Dufton, P. L., Hunter, I., Evans, C. J., Lennon, D. J., Smartt, S. J., \& Ryans, R. S. I. The VLT-FLAMES survey of massive stars: evolution of surface $\mathrm{N}$ abundances and effective temperature scales in the Galaxy and Magellanic Clouds. Astron. \& Astrophys., 471, 625-643 (2007)

44. Bouret, J.-C., Lanz, T., Hillier, D. J., Heap, S. R., Hubeny, I., Lennon, D. J., Smith, L. J., \& Evans, C. J. Quantitative Spectroscopy of O Stars at Low Metallicity: O Dwarfs in NGC 346. Astrophys. J., 595, 1182-1205 (2003)

45. Korn, A. J., Becker, S. R., Gummersbach, C. A., \& Wolf, B. Chemical abundances from Magellanic cloud B stars. Astron. \& Astrophys., 353, 655-665 (2000)

46. Luck, R. E., Moffett, T. J., Barnes, T. G., III, \& Gieren, W. P. Magellanic Cloud Cepheids Abundances. Astron. J., 115, 605-634 (1998) 
47. Jenkins, E. B. A Unified Representation of Gas-Phase Element Depletions in the Interstellar Medium. Astrophys. J., 700, 1299-1348 (2009)

48. Cartledge, S. I. B., Lauroesch, J. T., Meyer, D. M., \& Sofia, U. J. The Homogeneity of Interstellar Elemental Abundances in the Galactic Disk. Astrophys. J., 641, 327-346 (2006)

49. Savage, B. D., \& Sembach, K. R. Interstellar Abundances from Absorption-Line Observations with the Hubble Space Telescope. Ann. Rev. Astron. Astrophys., 34, 279-330 (1996)

50. Pequignot, D., \& Aldrovandi, S. M. V. The ionization balance in H I regions. Astron. \& Astrophys., 161, 169-176 (1986)

51. White, R. E. Interstellar lithium - Differential depletion in diffuse clouds. Astrophys. J., 307, 777-786 (1986)

52. Samland, M. Modeling the Evolution of Disk Galaxies. II. Yields of Massive Stars. Astrophys. $J .$, 496, 155-(1998)

53. Hunter, I., et al. The VLT-FLAMES survey of massive stars: constraints on stellar evolution from the chemical compositions of rapidly rotating Galactic and Magellanic Cloud B-type stars. Astron. \& Astrophys., 496, 841-853 (2009)

54. Dufton, P. L., Ryans, R. S. I., Trundle, C., Lennon, D. J., Hubeny, I., Lanz, T., \& Allende Prieto, C. B-type supergiants in the SMC: Chemical compositions and comparison of static and unified models. Astron. \& Astrophys., 434, 1125-1137 (2005)

55. Venn, K. A. A-Type Supergiant Abundances in the Small Magellanic Cloud: Probes of Evolution. Astrophys. J., 518, 405-421 (1999)

56. Peimbert, M., Peimbert, A., \& Ruiz, M. T. The Chemical Composition of the Small Magellanic Cloud H II Region NGC 346 and the Primordial Helium Abundance. Astrophys. J., 541, 688$700(2000)$

57. Zhang, H. W., Gehren, T., Butler, K., Shi, J. R., \& Zhao, G. Potassium abundances in nearby metal-poor stars. Astron. \& Astrophys., 457, 645-650 (2006) 
58. Takeda, Y., Zhao, G., Chen, Y.-Q., Qiu, H.-M., \& Takada-Hidai, M. On the Abundance of Potassium in Metal-Poor Stars. Pub. Astron. Soc. Japan, 54, 275-284 (2002)

59. Tolstoy, E., Hill, V., \& Tosi, M. Star-Formation Histories, Abundances, and Kinematics of Dwarf Galaxies in the Local Group. Ann. Rev. Astron. Astrophys., 47, 371-425 (2009)

60. Baade, D., Cristiani, S., Lanz, T., Malaney, R. A., Sahu, K. S., \& Vladilo, G. Reduced upper limits on the equivalent width of interstellar Li I 670.8 towards SN 1987 A. Astron. \& Astrophys., 251, 253-258 (1991)

61. Baade, D., \& Magain, P. Very low upper limits on the strength of interstellar lithium lines toward SN 1987 A. Astron. \& Astrophys., 194, 237-242 (1988)

62. Sahu, K. C., Pottasch, S. R., \& Sahu, M. Search for primordial lithium in the interstellar medium towards SN 1987A. Astron. \& Astrophys., 207, L1-(1988)

63. Vidal-Madjar, A., Andreani, P., Cristiani, S., Ferlet, R., Lanz, T., \& Vladilo, G. The interstellar spectrum toward SN 1987A. Astron. \& Astrophys., 177, L17-L20 (1987)

64. Sansonetti, C. J., Richou, B., Engleman, R., Jr., \& Radziemski, L. J. Measurements of the resonance lines of ${ }^{6} \mathrm{Li}$ and ${ }^{7} \mathrm{Li}$ by Doppler-free frequency-modulation spectroscopy. Phys. Rev. A, 52, 2682-2688 (1995)

65. Welty, D. E., Hobbs, L. M., \& Kulkarni, V. P. A high-resolution survey of interstellar NA I D1 lines. Astrophys. J., 436, 152-175 (1994)

66. Rosman, K. J. R., \& Taylor, P. D. P. Isotopic Compositions of the Elements 1997. Journal of Physical and Chemical Reference Data, 27, 1275-1287 (1998)

67. Knauth, D. C., Federman, S.R., Lambert, D.L., \& Crane, P. Newly Synthesized Lithium in the Interstellar Medium. Nature, 405 656-658 (2000)

68. Reeves, H., Fowler, W.A., \& Hoyle, F. Galactic Cosmic Ray Origin of Li, Be and B in Stars. Nature, 226, 727-729 (1970) 
69. Meneguzzi, M., Audouze, J., \& Reeves, H. The production of the elements Li, Be, B by galactic cosmic rays in space and its relation with stellar observations. Astron. \& Astrophys., 15, 337-359 (1971)

70. Steigman, G., \& Walker, T. P. Production of Li, Be, and B in the early Galaxy. Astrophys. J., 385, L13-L16 (1992)

71. Indriolo, N., Fields, B. D., \& McCall, B. J. The Implications of a High Cosmic-Ray Ionization Rate in Diffuse Interstellar Clouds. Astrophys. J., 694, 257-267 (2009)

72. Kalberla, P. M. W., Burton, W. B., Hartmann, D., Arnal, E. M., Bajaja, E., Morras, R., Pöppel, W. G. L. The Leiden/Argentine/Bonn (LAB) Survey of Galactic HI. Final data release of the combined LDS and IAR surveys with improved stray-radiation corrections. Astron. \& Astrophys., 440, 775-782 (2005)

73. Casuso, E., \& Beckman, J. E. Production of Lithium in the Galactic Disk. Pub. Astron. Soc. Pacific, 112, 942-960 (2000)

74. D’Antona, F., \& Matteucci, F. Galactic evolution of lithium. Astron. \& Astrophys., 248, 62-71 (1991)

75. Mathews, G. J., Alcock, C. R., \& Fuller, G. M. Galactic chemical evolution with low and high primordial lithium. Astrophys. J., 349, 449-457 (1990)

76. Woosley, S. E., Hartmann, D. H., Hoffman, R. D., \& Haxton, W. C. The $\nu$-process. Astrophys. $J .$, 356, 272-301 (1990) 